Research Article

\title{
Vibration Characteristics and Reduction Analysis of Lifting Pipe under Ocean Current
}

\author{
Qinghui Song $\mathbb{D},{ }^{1}$ Haiyan Jiang $\mathbb{D},{ }^{2}$ Qingjun Song $\mathbb{D}^{2},{ }^{2}$ Linjing Xiao $\mathbb{D}^{1},{ }^{1}$ and PeiSi Zhong $\mathbb{D}^{1}$ \\ ${ }^{1}$ Department of Mechanical and Electronic Engineering, Shandong University of Science \& Technology, Qingdao 264005, China \\ ${ }^{2}$ Tai-an School, Shandong University of Science \& Technology, Tai-an 271009, China \\ Correspondence should be addressed to Linjing Xiao; skd990278@sdust.edu.cn
}

Received 13 April 2021; Revised 15 June 2021; Accepted 17 June 2021; Published 5 July 2021

Academic Editor: Baoping Cai

Copyright ( 2021 Qinghui Song et al. This is an open access article distributed under the Creative Commons Attribution License, which permits unrestricted use, distribution, and reproduction in any medium, provided the original work is properly cited.

Effects of vibration absorbers on the lifting pipe in deep-sea mining have been attracting more and more attention in the recent three decades; however, there are very few reports about the influence of ocean current on the pipe vibration in the literature. Considering the geometrical features of the prototype, this paper establishes the physical model, mathematical model, and simulation model of the lifting subsystem. A comprehensive finite element model for the deep-sea mining system by the OrcaFlex is developed to explore the influences of buffer mass and sailing velocity on the deflection angle and the axial load and stress of the lifting pipe. Analytical and numerical simulations have been formulated to illustrate variation rule of longitudinal vibration and axial stress at the position of pump and buffer for the lifting subsystem to determine the dynamic vibration absorber parameters. In this paper, MATLAB and Orcaflex software are used to verify the mathematical model. The simulation results show that the attaching DVAs can effectively reduce the axial stress, and longitudinal displacement at certain positions of the lifting pipe.

\section{Introduction}

The development of renewable energy and the application of high and new technology need the support of new infrastructure that will consume a different mix of minerals from current applications, including not only "critical" metals such as rare earths, but also vast amounts of common commodities such as copper, steel, and cement [1]. The huge potential of marine mineral resources has been confirmed by many researchers, and deep ocean mining is being pushed to the next frontier of resource development [2]. Rich marine mineral resources will become irreplaceable resources for human development and utilization. Therefore, the development of marine mineral resources has become the strategic goal of all countries in the world. Companies in China, the UK, Belgium, Germany, France, and Japan have won contracts for exploration of deep-sea resources for three different mineral resources: seafloor massive sulphides, ferromanganese crusts, and poly metallic nodules [3]. In 2019, commercial deep-sea mining has begun off the coast of Papua New Guinea [4].
The solid mineral resources from the deep-sea bottom, such as ferromanganese nodules and cobalt-manganese crusts, most common ferromanganese nodules, are found typically at water depths of 4000-7000 m, and in the coastal areas of the shelf [5]. The deep-sea mining system consists of an ore collection system, a mother station, and a transportation system between the miner and mother station [6]. The transportation system is an important part of deep-sea mining system, which transports the collected manganese nodules from the miners to the mother station.

The research on the dynamic performance of the transportation system is of great significance to the design, performance evaluation, and control of the deep-sea mining system [7]. Under the influence of wind and waves in the complex marine environment, the mining vessel will produce surge, sway, heave, pitch, roll, and yaw motion. Obviously, the movement of the mining vessel will produce a motion excitation to the lifting pipe, which affects the reliability of the transportation system and the sea miner; especially the heave direction motion caused by ocean wave 
motion has a significant impact on the safety and stability of deep-sea mining system operation.

The longitudinal vibration of lifting pipe has been continuously researched and improved since deep-sea mining began to be studied. In order to reduce the longitudinal vibration of the lifting pipe, Aso et al. [8,9] proposed a vibration absorber composed of a mass, springs, and dampers to the pump module as well as the buffer, and gave an active control system to control the longitudinal vibration of the pipe string [10]; the resonance amplitude at the top of the lifting pipe is greatly reduced. Kobayashi and Aso [11] installed a linear and a non-linear spring supports on the upper end of the lifting pipe, respectively; they found that linear spring supports often increase the resonance frequency of the string and the resonance amplitude of the buffer, and reduce the maximum axial load of the string. Cui and Aso [12] systematically studied the longitudinal vibration of the lifting pipe by Galerkin method, and proposed a method to reduce the amplitude and axial load of the lifting pipe by using additional vibration damper and sectional lifting. Using the method of separating variables, Erol [13] studied the overdamping and underdamping modes of the hoisting system, respectively, and obtained the exact analytical solutions of free response and forced response of the system damping under the heave motion of the mining vessel.Yu and Liu established a comprehensive dynamic model of orbit terrain interaction to adapt to China's deepsea mining system, and the simulation analysis of the vertical reciprocating motion process was carried out [14]. Oh et al. [15] designed a coupling device with rotational freedom for deep-sea mining flexible pipe and analyzed the motion characteristics of the coupling device with multi-body dynamic simulation method and finite element method. Kim et al. [16] used the subsystem synthesis method to establish the motion equations of the vessel lifting pipe system and the flexible mining robot subsystem, respectively, which improves the efficiency of the solution. Lee et al. [17] compared the finite difference method with lumped mass and the substructure method from the field of flexible multi-body dynamics in the deep seabed mining system, and it was reported that the finite difference method with lumped mass is more efficient than the substructure method if the same number of elements was used in the model. Ma et al. [18] compared the continuous line bucket lifting system and the pipe lifting system in terms of the lifting efficiency, the energy consumption, and the profitability and pointed out that the mining depth and the solid mineral production rate of the pipe lifting system have a wider application range compared with the continuous line bucket system. Yang and Liu [19] proposed a measuring method for solid-liquid twophase flow in slurry pipeline for deep-sea mining based on differential capacitively coupled contactless conductivity detection. Yang and Liu [20] put forward a heave compensation system combined with a vibration absorber and accumulator, and the frequency domain characteristics and compensation rate of the system were analyzed and compared. Li et al. [21] used the discrete element method, computational fluid dynamics method, to study the solidliquid two-phase flow in the deep-sea elevator pump and obtained the pressure distribution on the suction surface and pressure surface and the distribution of particles in the pump under different rotating speeds. Liu et al. [22] used Euler Lagrange two-phase flow model to simulate the turbulent flow of solid-liquid two-phase in the pump and used Finnie wear model to predict the wear rate of hydraulic components of deep-sea mining pump under different flow rate, rotation speed, and particle concentration. Cho et al. [6] studied the performance of the deep-sea manganese nodule experimental miner, designed the ore guiding system composed of chassis structure, buoyancy, crushing system, electric control system, track, and sea miner, and analyzed the coupling relationship of the ore guiding system by using the axiomatic design method. Liu et al. $[23,24]$ analyzed the longitudinal vibration characteristics of lifting pipe under different working based on the finite element method. Song et al. [25] firstly used the Lagrangian method to establish the dynamic model of the rigid space stepped-pipe strings system and obtained the analytical solution by modal analysis method.

It can be seen from the above references that the current research on deep-sea mining system focuses on three aspects: mining sea miner $[6,16]$, pump set $[21,22]$, and lifting pipe $[8-14,18,23-25]$. The research on lifting pipe mainly focuses on two directions: reducing the longitudinal vibration of the lifting pipe with additional vibration damper [8-10], computer simulation with dynamic simulation method, and finite element analysis [14, 19].

At present, these researches are focused on the lifting pipe within one kilometer and did not consider that the lifting pipe will rotate at a certain angle when it is affected by the complex environmental load. However, most of the solid mineral resources in the deep sea occur in the depth of 4000-7000 meters [5]. Therefore, it is necessary to study the performance of the lifting system close to the actual sea depth. The goal of this paper is to offer a quantitative assessment of the influence of ocean current on the vibration characteristics of lifting pipe during deep-sea mining operation. Thus, according to the mechanism of deep-sea mining, physical model and analytical model of lifting system are designed. Second, the current velocity is divided into four cases by means of linear leastsquare approximate subsection method to solve the geometric nonlinear migration problem of ocean current, and the linear motion balance equation is deduced. Then, considering the effect of ocean current on the lifting subsystem, the dynamic models of the lifting subsystem with or without dynamic vibration absorber are established. Meanwhile, a finite element model for deep-sea mining with the OrcaFlex software is developed to simulate the dynamic responses of lifting pipe under the action of ocean current. Last, the dynamic model of lifting subsystem is verified by comparing the experimental curve and the simulation curve.

\section{Model Setup and Linear Approximate Calculation under Current Force}

2.1. Model Setup under Current Force. The physical model of the lifting system is a $1: 80$-scale prototype tested in National Ocean Technology Center of China, as shown in Figure 1. The mining vessel and lifting pipe in the model are 
approximate to the objective entity. The wave load of the deep-sea mining system is provided by the wave making system and the wave height sensor. The motion state of the mining vessel and the axial force of the lifting pipe are obtained by using pull pressure sensor, attitude sensor, signal processing module, and signal acquisition software. In this study, the influence of ocean current on lifting pipe and the vibration reduction analysis of dynamic vibration absorber are mainly considered.

Figure 2 shows the general model of the lifting pipe subsystem in deep-sea mining system under the action of ocean current, which is mainly composed of lifting pipe (four parts), lifting pumps (concentrated mass: $M_{1}$ ), and buffer (concentrated mass: $M_{2}$ ). The upper end of the lifting pipe is hinged with the mining vessel, the lower end of the lifting pipe is connected with the buffer, and the lifting pump set is installed at $L_{2}$ from the top of the lifting pipe. $\mathrm{L}_{S i}(i=1,2,3,4)$ is the segment length of the pipe sting in still water, and $L_{\mathrm{S} 1}=500 \mathrm{~m}, L_{\mathrm{S} 2}=1000 \mathrm{~m}$, $L_{\mathrm{S} 3}=2000 \mathrm{~m}, L_{\mathrm{S} 4}=3500 \mathrm{~m}, L_{\mathrm{S} 5}=5000 \mathrm{~m}$. The current forces brought to bear on each segment are $q_{N 1}, q_{N 2}, q_{N 3}$, $q_{N 4}$, and $q_{N 5}$. In addition, $g$ is the acceleration of gravity and $\gamma$ is the linear density of the lifting pipe.

As can be seen from Figure 2, the lifting pipe has four parts, the length of each part is $L_{i}$, and the basic parameters of lifting pipe are shown in Table 1.

\subsection{Linear Approximate Calculation under Ocean Current.} In order to solve the geometric nonlinear problem of current velocity, a linear approximate solution of current velocity in deep-sea mining is proposed in this paper. In this linear approximation model, the lifting pipe string is regarded as a rigid body. When the string is placed in still water, the twodimensional coordinate system $\mathrm{W}-\mathrm{O}-\mathrm{V}$ is established, and the $O-W$ axis is vertical downward, and the positive direction of $O-V$ axis is consistent with the wave propagation direction. When it is placed in ocean current, the string will shift angle $\theta$, and its coordinate system is $X-O-Y$.

The stability analysis of deep-sea mining lifting pipe involves ocean current, resistance coefficient, Reynolds number (Re), and other factors [26]. In particular, the velocity and direction of the ocean current vary significantly with depth and thus affect the dynamic characteristics of the lifting pipe. Using computer fitting methods, the current velocity is calculated depending on its depth below ocean surface [26]; one could obtain

$$
V_{c}=b+k \times\left(\frac{5000-x}{5000}\right)^{12},
$$

where $x$ is the depth below ocean surface, and the value is zero on the seawater surface. $b$ and $k$ are constants and coefficients related to current resistance and here we take 0.1 and 1.6 , respectively.
The fitting current velocity is shown as A-curve in Figure 3. Obviously, the current velocity becomes smaller and smaller with the deepening of the seawater depth and it no longer increases with the deepening of the seawater depth, but presents a steady flow when the seawater depth exceeds 2000 meters. Since the nonlinear complex models are generally difficult to solve, the current velocity $V_{C}$ can be linearized into four cases by means of linear least-square approximate method, and they can be expressed as

$$
\begin{cases}V_{C 1}=-\left(\frac{1.15}{500}\right) x+1.7, & 0 \leq x \leq L_{s 1}, \\ V_{C 2}=-\left(\frac{0.34}{500}\right) x+0.89, & L_{s 1} \leq x \leq L_{s 2}, \\ V_{C 3}=-\left(\frac{0.055}{500}\right) x+0.32, & L_{s 2} \leq x \leq L_{s 3}, \\ V_{C 4}=0.1, & L_{s 3} \leq x \leq L_{s 5} .\end{cases}
$$

Figure 3 shows a comparison of the fitting and linearized current velocity with ocean depth. The red-A line is the fitting current velocity curve, and the blue-B line is the current velocity after linearization. It can be clearly seen that the linearized curve is very close to the actual curve.

The current force per unit length acting on the lifting pipe can be expressed by Morison equation as

$$
f_{N}(x)=\left(\frac{1}{2}\right) C_{D} \rho_{w} D_{j}\left|V_{N}\right| V_{N},
$$

where $C_{\mathrm{D}}$ is the drag coefficient, $C_{\mathrm{D}}=1.2, \rho_{w}$ is the seawater density, $D_{j}(j=1,2,3,4)$ is the external diameter of the lifting pipe, and $V_{N}$ is normal current velocity of $V_{C}$.

According to the linearization of the current velocity in equation (2), the linearization of the normal current force on the lifting pipe can be expressed as follows:

$$
q_{N i}(x)=a_{i} x+b_{i}, i=1,2,3,4,
$$

where $a_{i}$ and $b_{i}$ are related to current velocity and current force per unit length of lifting pipe.

For $a_{i}$

$$
\left\{\begin{array}{l}
a_{1}=\frac{f_{N}\left(L_{s 1}\right)-f_{N}(0)}{1000} \\
a_{2}=\frac{f_{N}\left(L_{s 2}\right)-f_{N}\left(L_{s 1}\right)}{1000} \\
a_{3}=\frac{f_{N}\left(L_{s 3}\right)-f_{N}\left(L_{s 2}\right)}{1000} \\
a_{4}=a_{5}=0
\end{array}\right.
$$




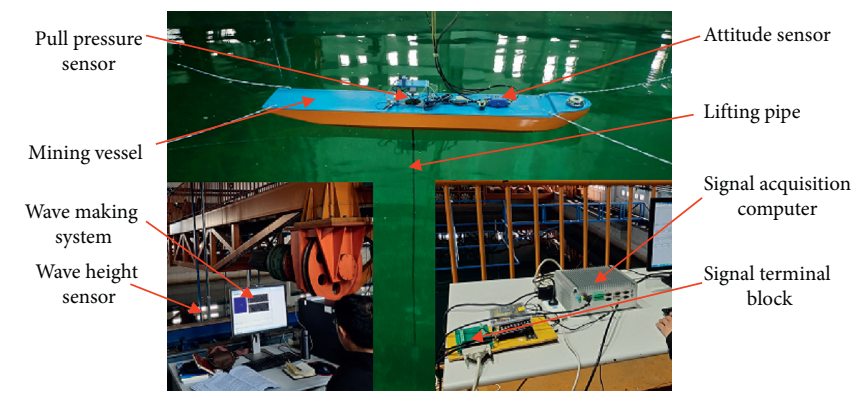

Figure 1: Physical model of the lifting subsystem.

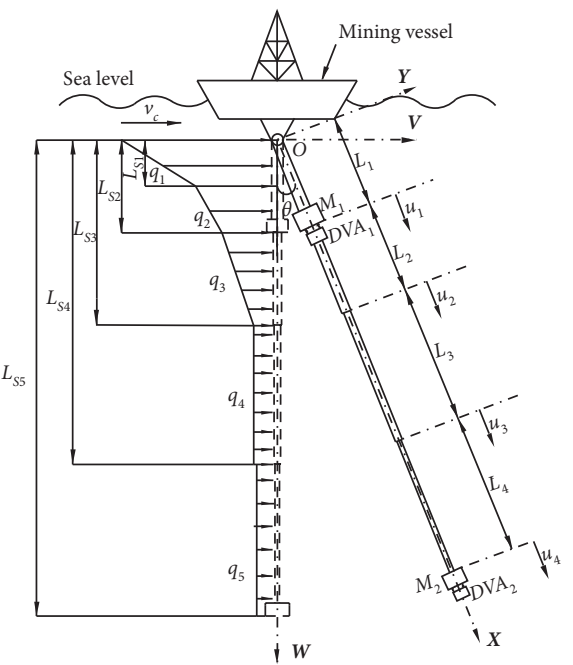

(a)
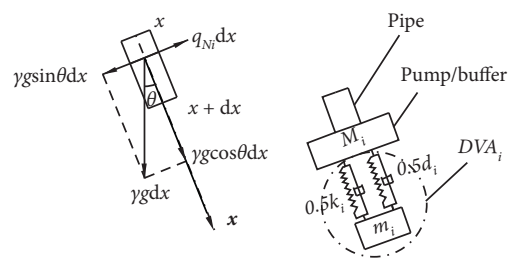

(b)

(c)

Figure 2: The mechanical model of the lifting pipe subsystem under the action of ocean current. (a) Mechanical model. (b) Forcemicro element. (c) DVA.

TABLE 1: Basic parameters of the lifting pipe subsystem.

\begin{tabular}{lccccc}
\hline Pipe section & Length $(\mathrm{m})$ & Internal diameter, $d(\mathrm{~mm})$ & External diameter, $D(\mathrm{~mm})$ & Wall thickness, $h(\mathrm{~mm})$ & Density, $\rho\left(\mathrm{kg} / \mathrm{m}^{3}\right)$ \\
\hline$L_{1}$ & 1000 & 206 & 254 & 24 & 17 \\
$L_{2}$ & 1000 & 206 & 240 & 13 & 7850 \\
$L_{3}$ & 1500 & 206 & 232 & 10 \\
$L_{4}$ & 1500 & 206 & 226 & 10 \\
\hline
\end{tabular}

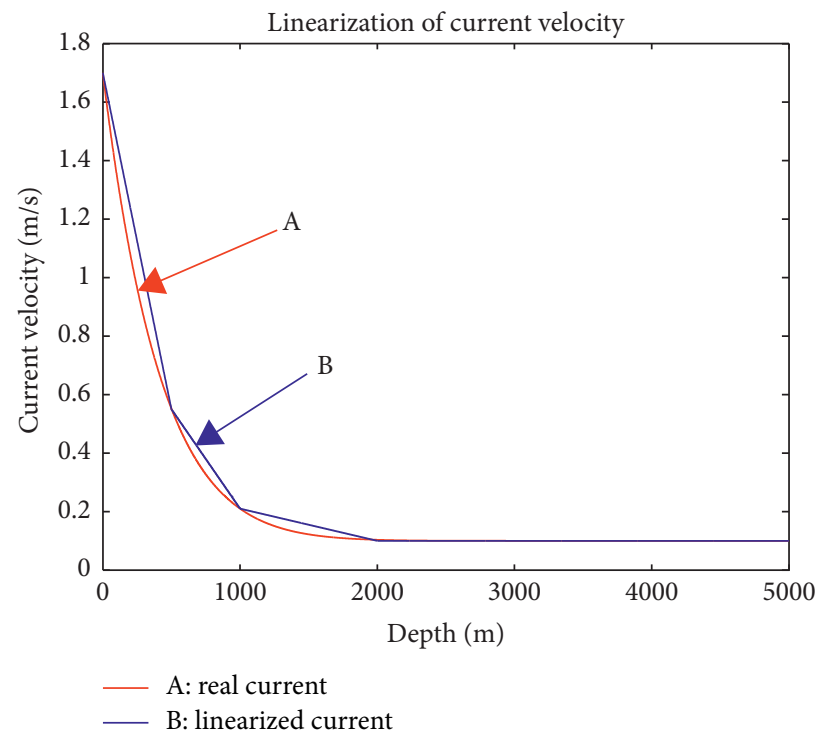

FIgURE 3: The current velocity curves below ocean surface. 
For $b_{i}$,

$$
\left\{\begin{array}{l}
b_{1}=f_{N}(0) \\
b_{2}=2 f_{N}\left(L_{s 2}\right)-f_{N}\left(L_{s 1}\right) \\
b_{3}=3 f_{N}\left(L_{s 2}\right)-2 f_{N}\left(L_{s 3}\right) \\
b_{4}=\left(\frac{(0.1-V)^{2} D_{3} C_{D} \rho_{w} \cos ^{2} \theta}{2}\right) \\
b_{5}=\left(\frac{(0.1-V)^{2} D_{4} C_{D} \rho_{w} \cos ^{2} \theta}{2}\right)
\end{array}\right.
$$

Under the action of ocean current, the lifting pipe will rigidly offset a certain angle $(\theta)$ around the origin $\mathbf{O}$ when the system is balanced. According to the moment balance condition at the origin, the equation of moment balance can be deduced as

$$
\begin{aligned}
& \frac{L_{s 1}}{3}\left(\int_{0}^{L_{s 1}} q_{N 1} \mathrm{~d} x-q_{N 1}^{*} L_{s 1}\right)+\left(\frac{q_{N 1}^{*} L_{s 1}^{2}}{2}\right)+\sum_{n=2}^{3}\left\{\frac{2 L_{s(n-1)}+L_{s n}}{3}\left[\int_{L_{s(n-1)}}^{L_{s n}} q_{N n} \mathrm{~d} x-q_{N n}^{*}\left(L_{s n}-L_{s(n-1)}\right)\right]+\left(\frac{L_{s n}^{2}-L_{s(n-1)}^{2}}{2}\right) q_{N n}^{*}\right\} \\
& \quad-\int_{0}^{L_{s 2}} \gamma_{1} g x \sin \theta \mathrm{d} x+\sum_{n=4}^{5} \frac{L_{s n}+L_{s(n-1)}}{2} \int_{L_{s(n-1)}}^{L_{s n}} q_{N n} \mathrm{~d} x-\sum_{n=2}^{4} \frac{L_{s n}+L_{s(n+1)}}{2} \int_{L_{s n}}^{L_{s(n+1)}} \gamma_{n} g \sin \theta \mathrm{d} x-\left[R_{M 1} L_{s 2}+R_{M 2} L_{s 5}\right] g \sin \theta=0
\end{aligned}
$$

where $q_{N i}^{*}$ is the unit load of $q_{N i}(x)$ in $L_{s i}$, that is, $q_{N i}^{*}=q_{N i}\left(L_{s i}\right)$. Here, the $R_{M 1}$ and $R_{M 2}$ are constants related to the quality, and $R_{M 1}=M_{1}, R_{M 2}=M_{2}$ when the pipe is not equipped with a vibration absorber.

\section{Vibration Analysis of the Lifting Subsystem without DVA}

3.1. Mathematical Model of Longitudinal Vibration. When a wave propagates forward, it moves sinusoidally and its shape remains unchanged [27], so the mining vessel is subjected to a harmonic motion with heave amplitude $\eta_{0}$ at frequency $\Omega$, represented as $\eta(t)=\eta_{0} e^{j \Omega t}$. In the $W-O-V$ coordinate system, the excitation motions of the $O V$ axis and the $O W$ axis can be represented by $\eta_{v}(t)=\eta_{0} e^{j \Omega t} \sin \theta$ and $\eta_{w}(t)=\eta_{0} e^{j \Omega t} \cos \theta$. When the lifting pipe is working, the mass per unit length of lifting pipe $\gamma_{i}$ is calculated by the following formula:

$$
\gamma_{i}=\frac{\pi\left(D_{i}^{2}-d_{i}^{2}\right) \rho}{4}-\left(\frac{\pi D_{i}^{2} \rho_{w}}{4}\right), \quad(i=1,2,3,4),
$$

where $D_{i}$ and $d_{i}$ are the external and internal diameters of each lifting pipe $L_{i}$, respectively. $\rho$ is the density of lifting pipe material, and $\rho_{w}$ is seawater density.

The mechanical model of the lifting subsystem is shown in Figure 2. The lifting pipe has four parts, the length of each part is $L_{i}$, the elastic modulus is $E_{i}$, the cross-sectional area is $S_{i}$, and the mass per unit length is $\gamma_{i}$. The viscous damping coefficient of the surrounding seawater is $c_{i}$, and the relevant physical parameters are given in Table 2.

The axial displacement of each section is usually different because the lifting pipe is composed of infinite particles. Therefore, the displacement of any section $x$ of the $i$-th pipe is a function of position and time, denoted as $u_{i}(x, t)$. The equations of motion of the lifting pipe can be established as

$$
E_{i} S_{i} \frac{\partial^{2} u_{i}}{\partial x^{2}}-\left(\gamma_{i} \frac{\partial^{2} u_{i}}{\partial t^{2}}-c_{i} \frac{\partial u_{i}}{\partial t}\right)=0, \quad i=1,2,3,4
$$

Set $b_{i}=\sum_{j=1}^{i} L_{j}(i=1,2,3,4)$; then the boundary and the continuity conditions of lifting subsystem in the $X-O-Y$ coordinate system can be formulated as

$$
\left.\begin{array}{l}
u_{1}(0, t)=\eta_{0} e^{j \Omega t} \cos \theta \\
u_{i}\left(b_{i}, t\right)=u_{i+1}\left(b_{i}, t\right) .
\end{array}\right\}
$$

The following equations are introduced to represent the force continuity condition:

$$
\left.\begin{array}{l}
\left.E_{1} S_{1} \frac{\partial u_{1}(x, t)}{\partial x}\right|_{x=b_{1}}+\left.M_{1} \frac{\partial^{2} u_{1}(x, t)}{\partial t^{2}}\right|_{x=b_{1}}=\left.E_{2} S_{2} \frac{\partial u_{2}(x, t)}{\partial x}\right|_{x=b_{1}}, \\
\left.E_{i} S_{i} \frac{\partial u_{i}(x, t)}{\partial x}\right|_{x=b_{i}}=\left.E_{i+1} S_{i+1} \frac{\partial u_{i+1}(x, t)}{\partial x}\right|_{x=b_{i}}, \quad i=2,3 \\
\left.E_{4} S_{4} \frac{\partial u_{4}(x, t)}{\partial x}\right|_{x=b_{4}}+\left.M_{2} \frac{\partial^{2} u_{4}(x, t)}{\partial t^{2}}\right|_{x=b_{4}}=0 .
\end{array}\right\}
$$


TABLE 2: Relevant physical parameters of the lifting pipe subsystem.

\begin{tabular}{ccccccc}
\hline$i$ & $L_{i}(\mathrm{~m})$ & $\gamma_{i}(\mathrm{~kg} / \mathrm{m})$ & $S_{i}\left(\mathrm{~m}^{2}\right)$ & $M_{i}(\mathrm{~kg})$ & $c_{i}(\mathrm{~N} \cdot \mathrm{s} / \mathrm{m})$ & 400 \\
\hline 1 & 1000 & 175.13 & 0.0173 & 8000 & 300 & 20600 \\
2 & 1000 & 120.27 & 0.0119 & - & 4000 & 400 \\
3 & 1500 & 90.32 & 0.0089 & - & 400 & 20600 \\
4 & 1500 & 68.53 & 0.0068 & & 20600 \\
\hline
\end{tabular}

The method of separating variables $u_{i}(x, t)=\phi_{i}(x) \times$ $q(t)$ is introduced, and equation (8) is transformed into the following form:

$$
\frac{E_{i} S_{i}}{\gamma_{i} \phi_{i}(x)} \frac{d^{2} \phi_{i}(x)}{d x^{2}}=\left(\frac{1}{q(t)} \frac{d^{2} q(t)}{d t^{2}}+\frac{c_{i}}{\gamma_{i} q(t)} \frac{d q(t)}{d t}\right)=a_{i},
$$

where $a_{i}$ are complex constants to be determined.

Letting $q(t)$ in equation (11) be represented by $e^{\lambda t}$, here, $\lambda$ is an eigenvalue of vibration differential equation; then equation (11) takes the form

$$
\left\{\begin{array}{l}
\frac{d^{2} \phi_{i}(x)}{d x^{2}}-\frac{\gamma_{i}}{E_{i} S_{i}} a_{i} \phi_{i}(x)=0, \quad i=1,2,3,4 \\
\lambda^{2}+\frac{c_{i}}{\gamma_{i}} \lambda=a_{i}, \quad i=1,2,3,4
\end{array}\right.
$$

Setting $v_{i}^{2}=\left(\gamma_{i} / E_{i} S_{i}\right) a_{i}$, the first equation in the above equation (12) is expressed as

$$
\frac{\mathrm{d}^{2} \phi_{i}(x)}{\mathrm{d} x^{2}}-v_{i}^{2} \phi_{i}(x)=0, \quad i=1,2,3,4 .
$$

Suppose that the general solution of equation (13) is expressed as

$$
\phi_{i}(x)=\left(\bar{A}_{i} e^{v_{i} x}+\bar{B}_{i} e^{-v_{i} x}\right), \quad i=1,2,3,4,
$$

where $\bar{A}_{i}$ and $\bar{B}_{i}$ are complex constants to be determined; the equation is equivalently expressed by the following equation:

$$
\left(\overline{A_{1}}+\overline{B_{1}}\right) e^{\lambda t}=\eta_{0} e^{j \Omega t} \cos \theta .
$$

According to the theory of solution of differential equation and [13], $\lambda$ must be equal to $j \Omega(\lambda=j \Omega)$. The boundary and the force continuity conditions given in equations (9)-(10) are transformed into the following forms as

$$
\left\{\begin{array}{l}
\phi_{1}\left(b_{i}, t\right)=\phi_{i+1}\left(b_{i}, t\right) \\
E_{1} S_{1} \frac{d \phi_{1}\left(b_{1}\right)}{d x} M_{1} \phi_{1}\left(b_{1}\right) \lambda^{2}=E_{2} S_{2} \frac{d \phi_{2}\left(b_{1}\right)}{d x} \\
E_{i} S_{i} \frac{d \phi_{i}\left(b_{i}\right)}{d x}=E_{i} S_{i+1} \frac{d \phi_{i+1}\left(b_{i}\right)}{d x}, \quad i=2,3 \\
E_{4} S_{4} \frac{d \phi_{4}\left(b_{4}\right)}{d x} M_{2} \phi_{4}\left(b_{4}\right) \lambda^{2}=0 .
\end{array}\right.
$$

Substituting equation (14) and the substitution of the expression $\lambda=j \Omega$ into equations (16) and (15) lead to

$$
\begin{aligned}
\mathbf{G} \times & {\left[\overline{A_{1}}, \overline{B_{1}}, \overline{A_{2}}, \overline{B_{2}}, \overline{A_{3}}, \overline{B_{3} A_{4}}, \overline{B_{4}}\right]^{T} } \\
& =\left[\eta_{0} \cos \theta, 0,0,0,0,0,0,0\right]^{T},
\end{aligned}
$$

where

$$
\mathbf{G}=\left[\begin{array}{cccccccc}
1 & 1 & 0 & 0 & 0 & 0 & 0 & 0 \\
e^{v_{1} b_{1}} & e^{-v_{1} b_{1}} & -e^{v_{2} b_{1}} & -e^{-v_{2} b_{1}} & 0 & 0 & 0 & 0 \\
\alpha_{1} e^{v_{1} b_{1}} & \beta_{1} e^{-v_{1} b_{1}} & -\kappa_{2} e^{v_{2} b_{1}} & \kappa_{2} e^{-v_{2} b_{1}} & 0 & 0 & 0 & 0 \\
0 & 0 & e^{v_{2} b_{2}} & e^{-v_{2} b_{2}} & -e^{v_{3} b_{2}} & -e^{-v_{3} b_{2}} & 0 & 0 \\
0 & 0 & \kappa_{2} e^{v_{2} b_{2}} & -\kappa_{2} e^{-v_{2} b_{2}} & -\kappa_{3} e^{v_{3} b_{2}} & \kappa_{3} e^{-v_{3} b_{2}} & 0 & 0 \\
0 & 0 & 0 & 0 & e^{v_{3} b_{3}} & e^{-v_{3} b_{3}} & -e^{v_{4} b_{3}} & -e^{-v_{4} b_{3}} \\
0 & 0 & 0 & 0 & \kappa_{3} e^{v_{3} b_{3}} & -\kappa_{3} e^{-v_{3} b_{3}} & -\kappa_{4} e^{v_{4} b_{3}} & \kappa_{4} e^{-v_{4} b_{3}} \\
0 & 0 & 0 & 0 & 0 & 0 & \alpha_{4} e^{v_{4} b_{4}} & \beta_{4} e^{-v_{4} b_{4}}
\end{array}\right] .
$$


Note that the coefficients $\alpha_{i}, \beta_{i}$, and $\kappa_{i}$ are the expressions of $M_{i}, E_{i}, S_{i}, v_{i}$, and $\lambda$, as follows:

$$
\left\{\begin{array}{l}
\alpha_{1}=M_{1} \lambda^{2}+E_{1} S_{1} v_{1}, \\
\alpha_{4}=M_{2} \lambda^{2}+E_{4} S_{4} v_{4}, \\
\beta_{1}=M_{1} \lambda^{2}-E_{1} S_{1} v_{1}, \\
\beta_{4}=M_{2} \lambda^{2}-E_{4} S_{4} v_{4}, \\
\kappa_{i}=E_{i} S_{i} v_{i}, \quad i=2,3,4 .
\end{array}\right.
$$

Program (code) was written in MATLAB R2013a environment to model the longitudinal vibration of lifting pipe in the deep-sea mining system using equations (8) to (17). The physical parameters required in the model are listed in Table 2, and the calculation results in equation (17) are shown in Table 3 , where $j$ is the imaginary unit. Therefore, the general solution of equation (13) $\phi_{i}(x)$ and the forced longitudinal displacements of lifting pipe $u_{i}(x, t)$ are determined.

3.2. Influence of Ocean Current on the Displacement of the Lifting Pipe. This paper mainly studies the influence of marine environment on the vibration of the lifting subsystem. Orcaflex software is based on the finite element method, combined with the time integration scheme to express the dynamic stress and response. OrcaFlex software allows modeling of waves, wind, and current which can be added to the lifting subsystem simulation. The inertia characteristics of the model were calculated in SolidEdge (a 3D CAD modeling tool) and were input into Orcaflex model of deep-sea mining system, as shown in Figure 4(a). The motions are simulated numerically using OrcaFlex under sea condition of level 6 operation, and the heave motion of the mining vessel is evaluated using Response Amplitude Operator (RAO). The ocean current parameter setting is shown in Figure 4(b), and the heave motion RAO curve of the mining vessel under the action of facing wave $\left(180^{\circ}\right)$ is shown in Figure 4(c).

Assuming that the water depth of deep-sea mining operation is $5000 \mathrm{~m}$, and it works under the sea condition of level 6 (wave height is $7 \mathrm{~m}$; wave period is $10 \mathrm{~s}$ ), according to heave motion RAO curve in Figure 4(c), the heave amplitude of mining vessel is $0.9 \mathrm{~m}$. In combination with $[12,15]$, the heave motion of the mining vessel can be simplified as a simple harmonic motion with the amplitude $\eta_{0}=1 \mathrm{~m}$ and the angular frequency $\Omega=0.6283 \mathrm{rad} / \mathrm{s}$. Considering the influence of ocean current on lifting pipe, according to the previous equations (3)-(6), the model of lifting subsystem is established and solved in MATLAB, and the deflection angle $\theta$ of the lifting pipe under the action of current is $0.5709^{\circ}$. Figure 5 shows longitudinal and lateral displacement of lifting pipe for harmonic motion of $e^{j 0.6283 t}$ with MATLAB.

It can be seen, with the increase of the length of the lifting pipe, the longitudinal vibration displacement also increases. If the influence of ocean current is not taken into account, the maximum longitudinal vibration displacement at the end of the lifting pipe is $16.75 \mathrm{~m}$; if the influence of current is taken into account, the maximum longitudinal vibration displacement at the end of the lifting pipe is $17.05 \mathrm{~m}$, which shows that the influence of current on the longitudinal vibration displacement of the lifting pipe is small. However, the current has a great influence on the lateral displacement of the lifting pipe, and the end displacement is as high as $49.82 \mathrm{~m}$, which will affect the operation of the sea miner. In summary, the ocean current has little influence on the longitudinal vibration of the lifting pipe, but it has great influence on its lateral displacement, which is enough to affect the normal operation of the sea miner. Therefore, some measures have to be taken to reduce this influence.

The Orcaflex software is used to calculate the longitudinal vibration displacement of lifting pipe at $1000 \mathrm{~m}$, $2000 \mathrm{~m}, 3500 \mathrm{~m}$, and $5000 \mathrm{~m}$ deep sea, as shown in Figure 6(a), and the corresponding longitudinal vibration curve is shown in Figure 6(b). At the same time, MATLAB is used to solve the differential equation of the lifting subsystem, and its longitudinal vibration displacement is shown in Figure 7. The comparison of longitudinal vibration simulation results is shown in Figure 8.

By carefully comparing Figures $6-8$, it is not difficult to find that the numerical simulation results in MATLAB and Orcaflex show good agreement, and the longer the lifting pipe, the greater the longitudinal displacement. In addition, Table 4 lists the longitudinal vibration displacement errors at different times and locations. It was obvious that the calculating results obtained by MATLAB are in accordance with the simulation results obtained by Orcaflex finite element model, and the longitudinal amplitude error at the end of the lifting pipe is the largest, only $5.07 \%$.

3.3. Axial Stress of the Lifting Pipe under Current Force. The total axial stress of the lifting pipe is composed of the gravity stress caused by its own weight and the dynamic stress caused by the vibration of the mining vessel.

The total axial stress of the lifting pipe is determined by the structure, elastic modulus, cross-sectional area, linear weight, linear damping coefficient, and other factors.

Using mechanical knowledge, the gravity stress of each stepped lifting pipe can be calculated as 
TABLe 3: Calculation values of $\bar{A}_{i}$ and $\bar{B}_{i}$ in equation (17) under ocean current.

\begin{tabular}{ccccc}
\hline$i$ & 1 & 2 & 3 & 4 \\
\hline $\bar{A}_{i}$ & $-0.3230+0.0648 \mathrm{j}$ & $-0.0103+0.0030 \mathrm{j}$ & $0.0001-0.0001 \mathrm{j}$ & $-0.0005+0.0002 \mathrm{j}$ \\
$\bar{B}_{i}$ & $1.8180-0.0648 \mathrm{j}$ & $1.7687-0.8299 \mathrm{j}$ & $2.6000-0.5900 \mathrm{j}$ & $4.3810+0.7707 \mathrm{j}$
\end{tabular}

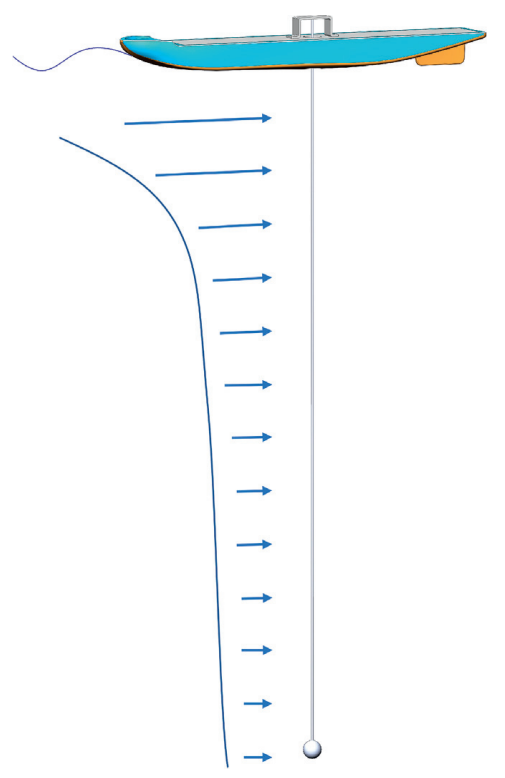

(a)

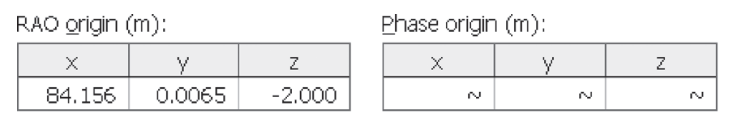

\begin{tabular}{|l|l|l|l|l|l|l|l|}
\hline $0^{\circ}$ & $45^{\circ}$ & $90^{\circ}$ & $135^{\circ}$ & $180^{\circ}$ & $225^{\circ}$ & $270^{\circ}$ & $315^{\circ}$ \\
\hline
\end{tabular}

Periods: $20-\frac{-1}{*}$

\begin{tabular}{|c|c|c|c|c|c|c|}
\hline \multirow[b]{2}{*}{$\begin{array}{l}\text { Periad } \\
\text { (s) }\end{array}$} & \multicolumn{2}{|c|}{ Surge } & \multicolumn{2}{|c|}{ Sway } & \multicolumn{2}{|c|}{ Heave } \\
\hline & $\begin{array}{l}\text { Ampl. } \\
(\mathrm{kN} / \mathrm{m})\end{array}$ & $\begin{array}{l}\text { Phase } \\
\text { (deg) }\end{array}$ & $\begin{array}{l}\text { Ampl. } \\
\text { (kN/m) }\end{array}$ & $\begin{array}{l}\text { Phase } \\
\text { (deg) }\end{array}$ & $\begin{array}{l}\text { Ampl. } \\
(\mathrm{kN} / \mathrm{m})\end{array}$ & $\begin{array}{l}\text { Phase } \\
\text { (deg) }\end{array}$ \\
\hline 2.03 & 21,600 & 110.7 & 2.070 & 137.4 & 28.900 & 284.3 \\
\hline 2.14 & 36.200 & 207.9 & 1.740 & 37.3 & 40.800 & 4.5 \\
\hline 2.26 & 53.700 & 290.5 & 3.350 & 11.2 & 213.00 & 353.4 \\
\hline 2.39 & 88.200 & 336.3 & 1.200 & 256.7 & 286.00 & 333.4 \\
\hline 2.55 & 114.00 & 308.3 & 2.330 & 239.6 & 448.00 & 277.2 \\
\hline 2.72 & 611.00 & 293.3 & 11.300 & 289.9 & 502.00 & 199.0 \\
\hline 2.92 & 114,00 & 5.2 & 5.550 & 288.5 & 661.00 & 91.4 \\
\hline 3.15 & 126.00 & 342.1 & 10.900 & 90.4 & 962.00 & 302.9 \\
\hline 3.42 & 209.00 & 197.5 & 0.284 & 144.5 & 890.00 & 195.1 \\
\hline 3.74 & 216.00 & 53.9 & 0.398 & 297.3 & 1050.0 & 41.4 \\
\hline 4.13 & 173.00 & 245.2 & 0.903 & 115.2 & 1110.0 & 228.1 \\
\hline 4.61 & 184.00 & 49.0 & 1.780 & 351.4 & 1330.0 & 37.9 \\
\hline
\end{tabular}

(b)

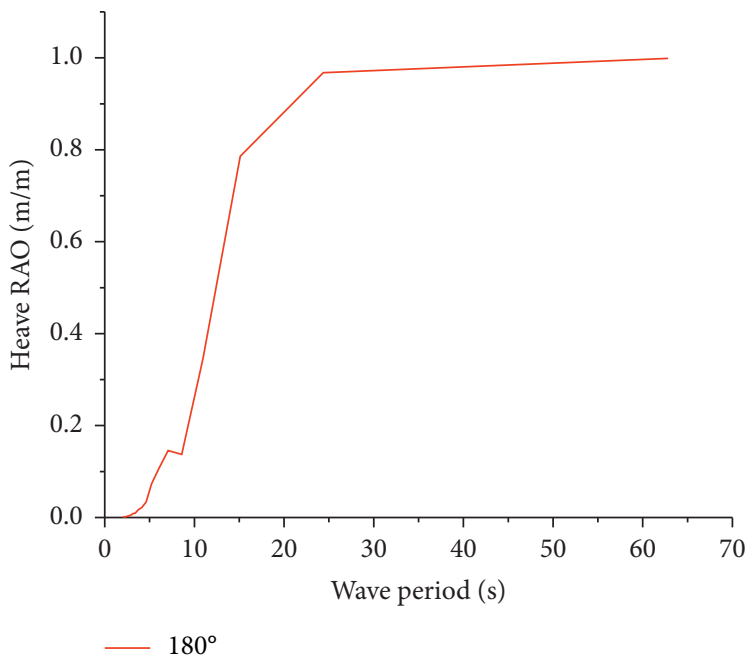

(c)

FIGURE 4: Simulation model based on Orcaflex: (a) solid edge model, (b) ocean current parameter setting, and (c) heave motion RAO curve. 


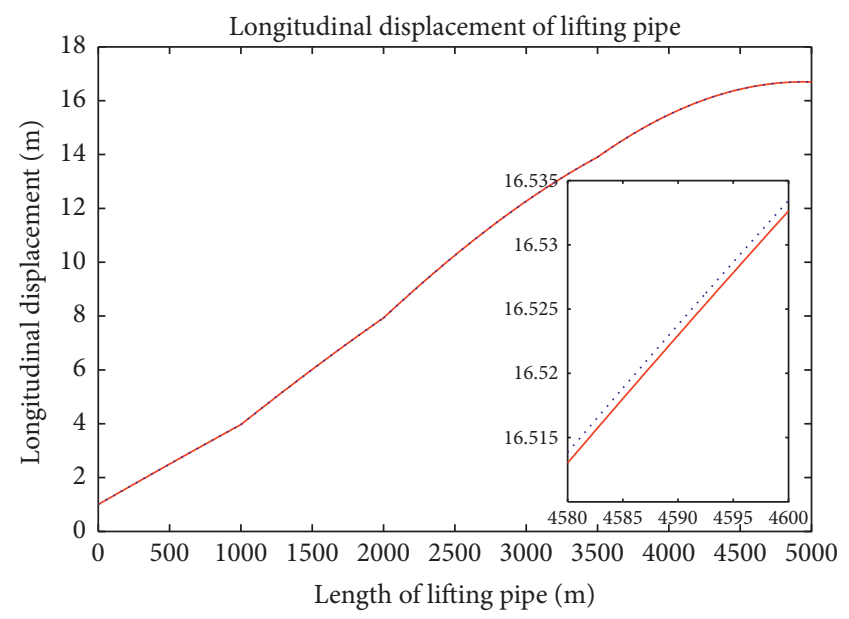

Without ocean current

With ocean current

(a)

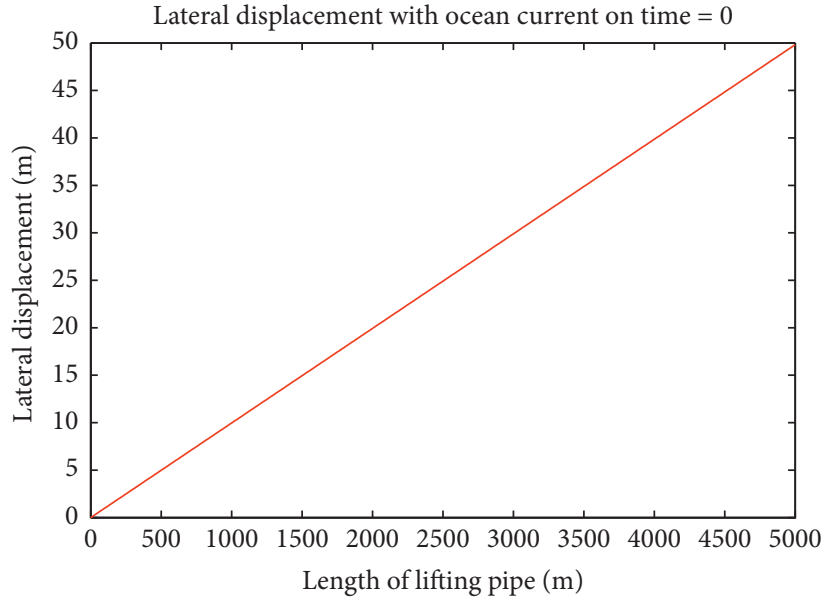

(b)

Figure 5: Longitudinal and lateral displacement of lifting pipe. (a) Longitudinal displacement. (b) Lateral displacement.

Modified $12: 17$ on $2021 / 3 / 20$ by OrcaFlex 10.0 e

Column A: Time (s)

Column B: line1 dynamic $z(\mathrm{~m})$ at $1000 \mathrm{~m}$

Column C: line1 dynamic $z(\mathrm{~m})$ at $2000 \mathrm{~m}$

Column D: line1 dynamic $z(\mathrm{~m})$ at $3500 \mathrm{~m}$

Column E: line1 dynamic $z(\mathrm{~m})$ at $5000 \mathrm{~m}$

Period: $t=0.000$ to $30.000 \mathrm{~s}$

\begin{tabular}{|l|c|c|c|c|}
\hline Column A & Column B & Column C & Column D & Column E \\
\hline 0.0 & 3.8999 & 7.5398 & 12.9997 & 16.1196 \\
0.1 & 3.7959 & 7.3388 & 12.6530 & 15.6898 \\
0.2 & 3.6789 & 7.1126 & 12.2630 & 15.2061 \\
0.3 & 3.5503 & 6.8639 & 11.8343 & 14.6745 \\
0.4 & 3.4112 & 6.5950 & 11.3707 & 14.0997 \\
0.5 & 3.2625 & 6.3076 & 10.8752 & 13.4852 \\
0.6 & 3.1049 & 6.0029 & 10.3498 & 12.8337 \\
0.7 & 2.9389 & 5.6820 & 9.7965 & 12.1476 \\
0.8 & 2.7652 & 5.3460 & 9.2173 & 11.4295
\end{tabular}

(a)

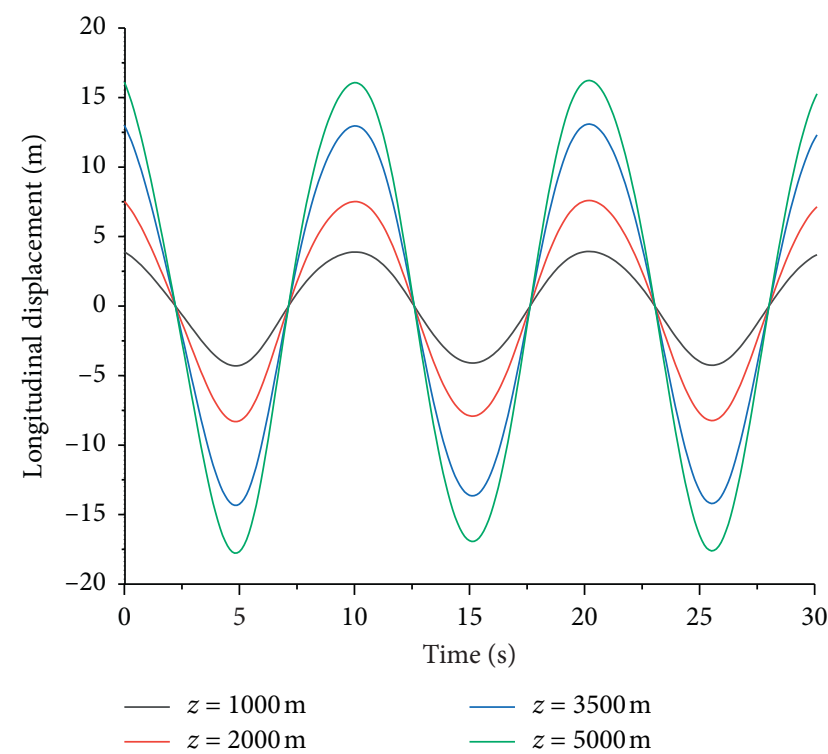

(b)

FiguRE 6: Simulation results of longitudinal vibration displacement based on Orcaflex: (a) calculation results and (b) longitudinal vibration curve. 


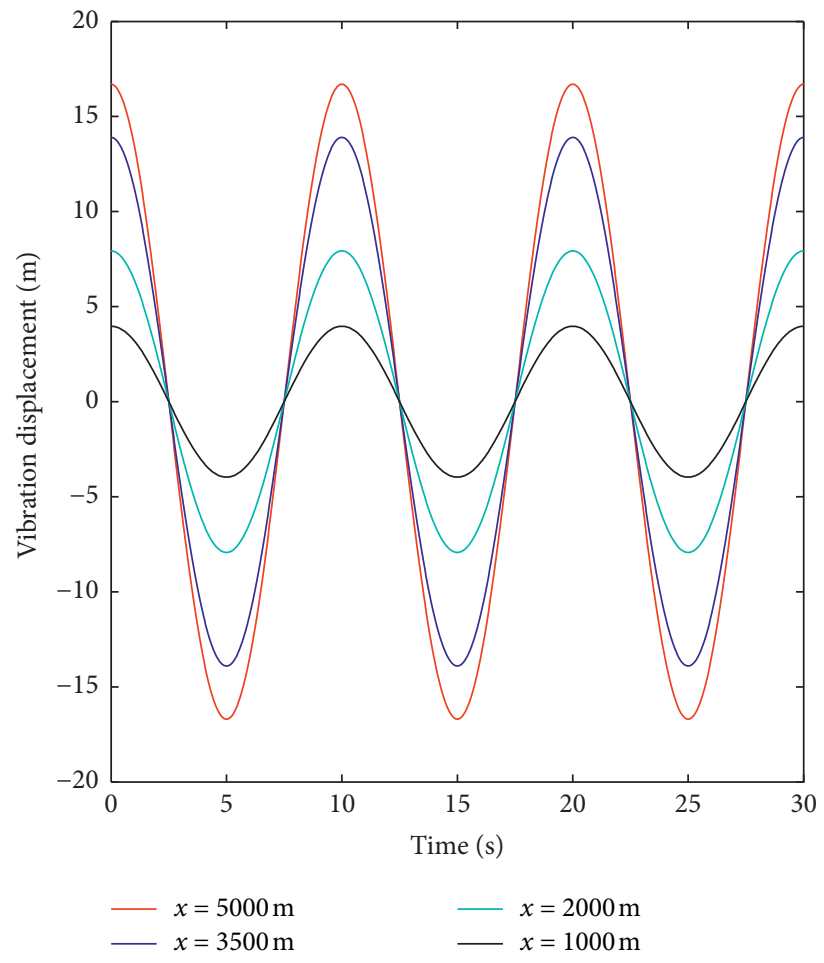

Figure 7: Longitudinal vibration displacement based on MATLAB.

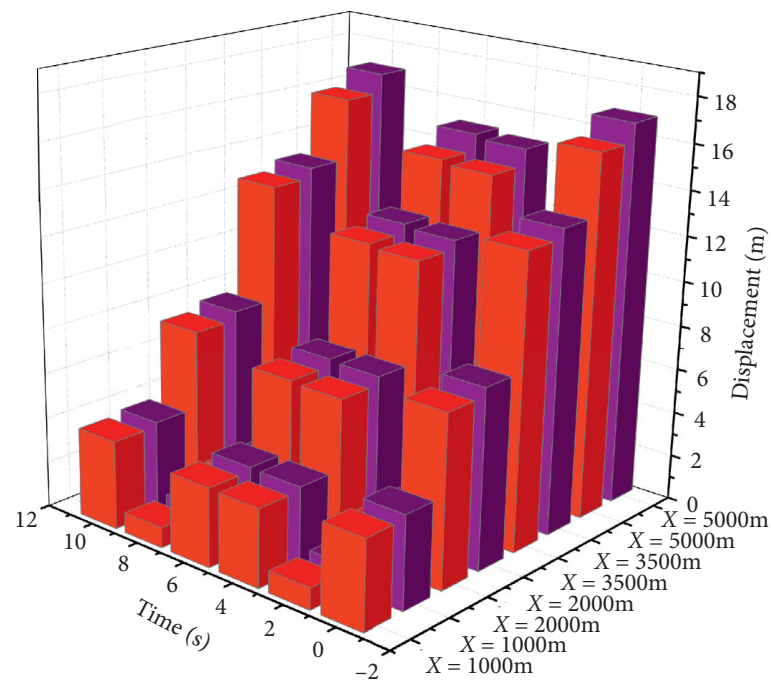

OrcaFlex

MATLAB

Figure 8: Comparison of longitudinal vibration simulation results. 
TABLE 4: Longitudinal vibration displacements of lifting pipe at different positions.

\begin{tabular}{lcccccccc}
\hline \multirow{2}{*}{ Time $(\mathrm{s})$} & \multicolumn{2}{c}{ At $1000 \mathrm{~m}(\mathrm{~m})$} & \multicolumn{2}{c}{ At $2000 \mathrm{~m}(\mathrm{~m})$} & \multicolumn{2}{c}{ At $3500 \mathrm{~m}(\mathrm{~m})$} & \multicolumn{2}{c}{ At $5000 \mathrm{~m}(\mathrm{~m})$} \\
& OrcaFlex & MATLAB & OrcaFlex & MATLAB & OrcaFlex & MATLAB & OrcaFlex & MATLAB \\
\hline 0 & 3.8999 & 4.0727 & 7.5398 & 7.8964 & 12.9997 & 13.4222 & 16.1196 \\
2 & 0.9537 & 0.996 & 1.8438 & 1.931 & 3.179 & 3.2823 & 3.942 \\
4 & -3.4231 & -3.5748 & -6.618 & -6.931 & -11.4104 & -11.7812 & -14.1488 & -14.9369 \\
6 & -3.4673 & -3.6209 & -6.7035 & -7.0206 & -11.5578 & -11.9334 & -14.3317 & -15.0583 \\
8 & 0.8926 & 0.9321 & 1.7257 & 1.8073 & 2.9753 & 3.072 & 3.6893 \\
Error & \multicolumn{2}{c}{$4.43 \%$} & \multicolumn{2}{c}{$4.73 \%$} & & 3.25 & & 3.07 \\
\hline
\end{tabular}

$$
\left.\begin{array}{ll}
\sigma_{g 1}(x, t)=\frac{M_{1}+M_{2}+\gamma_{1}\left(L_{1}-x\right)+\left(\gamma_{2} L_{2}+\gamma_{3} L_{3}+\gamma_{4} L_{4}\right)}{S_{1}} g \cos \theta, & 0 \leq x \leq L, \\
\sigma_{g 2}(x, t)=\frac{M_{2}+\gamma_{2}\left(L_{1}+L_{2}-x\right)+\left(\gamma_{3} L_{3}+\gamma_{4} L_{4}\right)}{S_{2}} g \cos \theta, & L_{1} \leq x \leq L_{1}+L_{2}, \\
\sigma_{g 3}(x, t)=\frac{M_{2}+\gamma_{3}\left(L_{1}+L_{2}+L_{3}-x\right)+\gamma_{4} L_{4}}{S_{3}} g \cos \theta, & L_{1}+L_{2} \leq x \leq L_{1}+L_{2}+L_{3}, \\
\sigma_{g 4}(x, t)=\frac{M_{2}+\gamma_{4}\left(L_{1}+L_{2}+L_{3}+L_{4}-x\right)}{S_{4}} g \cos \theta, & \\
\end{array}\right\}
$$

Under the action of oceanic wave $\eta(w, t)$, the lifting pipe and the mining vessel heave at the same frequency, so the dynamic stress on the lifting pipe is only related to the longitudinal displacement $u_{i}(x, t)$, and then the axial dynamic stress produced in the pipe can be obtained by the following equation:

$$
\sigma_{d i}(x, t)=E \frac{\partial u_{i}(x, t)}{\partial x} .
$$

\section{Vibration Analysis of the Lifting Subsystem with DVA}

In the complex marine environment, the vibration and axial stress of the lifting subsystem caused by the motion of the mining vessel are easy to cause the fatigue failure and failure of the subsystem. Under the action of ocean current, with the increase of the length of the lifting pipe, the lateral displacement at different positions of the pipe increases significantly, which seriously affects the safety performance of the lifting pipe. By adding vibration absorber, the vibration amplitude can be reduced by changing the natural frequency of the lifting subsystem, so as to reduce the dynamic maximum axial stress and the lateral displacement of the pipeline. This dynamic vibration absorber model consists of a spring element $k_{i}$, a damping element $d_{i}$, and absorber concentrated mass $m_{i}$, as shown in Figure 2(c). In this model, the pump and the buffer of the lifting pipe are equipped with a dynamic vibration absorber, respectively.

In this section, the calculation will be demonstrated by using the DVA physical parameter given in Table 5 and the previous parameters given in Tables 1 and 2 .

Applying the variable-separating method, let $z_{i}(x, t)=Z_{i}(x) \times q_{i}(t), \quad i=1,2$, be the displacement function of DVA; the motion differential equation of the first DVA can be calculated as

$$
\begin{aligned}
& m_{1} \frac{\partial^{2} z_{1}(x, t)}{\partial t^{2}}+k_{1}\left[z_{1}(x, t)-u_{1}\left(b_{1}, t\right)\right] \\
& +d_{1}\left[\frac{\partial z_{1}(x, t)}{\partial t}-\left.\frac{\partial u_{1}(x, t)}{\partial t}\right|_{x=b_{1}}\right]=0 .
\end{aligned}
$$

The second DVA's motion differential equation is as follows:

$$
\begin{aligned}
& m_{2} \frac{\partial^{2} z_{2}(x, t)}{\partial t^{2}}+k_{2}\left[z_{2}(x, t)-u_{4}\left(b_{4}, t\right)\right] \\
& \quad+d_{2}\left[\frac{\partial z_{2}(x, t)}{\partial t}-\left.\frac{\partial u_{4}(x, t)}{\partial t}\right|_{x=b_{4}}\right]=0 .
\end{aligned}
$$

When the two DVAs are equipped in pump and buffer respectively, the mathematical model of lifting subsystem can be established as 
TABle 5: Physical parameters of DVA.

\begin{tabular}{lccc}
\hline$i$ & $m_{i}(\mathrm{~kg})$ & $k_{i}(\mathrm{~N} / \mathrm{m})$ & $d_{i}(\mathrm{~N} \cdot \mathrm{s} / \mathrm{m})$ \\
\hline 1 & 1660 & 119000 & 200 \\
2 & 2600 & 119000 & 200 \\
\hline
\end{tabular}

$$
\left.\begin{array}{l}
\left.E_{1} S_{1} \frac{d \phi_{1}(x)}{d x}\right|_{x=b_{1}}+M_{1} \phi_{1}\left(b_{1}\right) \lambda^{2}+m_{1} Z_{1}(x) \lambda^{2}=\left.E_{2} S_{2} \frac{d \phi_{2}(x)}{d x}\right|_{x=b_{1}}, \\
m_{1} Z_{1}(x) \lambda^{2}+d_{1} \lambda\left[Z_{1}(x)-\phi_{1}\left(b_{1}\right)\right]+k_{1}\left[Z_{1}(x)-\phi_{1}\left(b_{1}\right)\right]=0, \\
\left.E_{4} S_{4} \frac{d \phi_{4}(x)}{d x}\right|_{x=b_{4}}+M_{2} \phi_{4}\left(b_{4}\right) \lambda^{2}+m_{2} Z_{2}(x) \lambda^{2}=0, \\
m_{2} Z_{2}(x) \lambda^{2}+d_{2} \lambda\left[Z_{2}(x)-\phi_{4}\left(b_{4}\right)\right]+k_{2}\left[Z_{2}(x)-\phi_{4}\left(b_{4}\right)\right]=0 .
\end{array}\right\}
$$

Substituting the form of complex solution of differential equation into the second equation in equation (24), the displacement expression of DVA can be obtained as follows:

$$
\left.\begin{array}{l}
Z_{1}(x)=\overline{A_{1}} \frac{d_{1} \lambda+k_{1}}{m_{1} \lambda^{2}+d_{1} \lambda+k_{1}} e^{v_{1} b_{1}}+\overline{B_{1}} \frac{d_{1} \lambda+k_{1}}{m_{1} \lambda^{2}+d_{1} \lambda+k_{1}} e^{-v_{1} b_{1}}, \\
Z_{2}(x)=\overline{A_{4}} \frac{d_{2} \lambda+k_{2}}{m_{2} \lambda^{2}+d_{2} \lambda+k_{2}} e^{v_{4} b_{4}}+\overline{B_{4}} \frac{d_{2} \lambda+k_{2}}{m_{2} \lambda^{2}+d_{2} \lambda+k_{2}} e^{-v_{4} b_{4}} \cdot
\end{array}\right\}
$$

When the buffer and pump are equipped with DVA, respectively, $R_{M 1}=M_{1}+m_{1}, R_{M 2}=M_{2}+m_{2}$ in equation (6). Numerical simulation has been performed with MATLAB to determine that the deflection angle $\theta$ of the lifting pipe is $0.4307^{\circ}$. Combining equations (14) and (24), the equation for determining the unknown quantity is obtained as equation (17). In the case of dynamic vibration absorbers, the coefficients $\alpha_{1}, \alpha_{4}, \beta_{1}$, and $\beta_{4}$ in equation (18) are recombined as follows:

$$
\left.\begin{array}{l}
\alpha_{1}=\left(M_{1}+\frac{m_{1}\left(d_{1} \lambda+k_{1}\right)}{m_{1} \lambda^{2}+d_{1} \lambda+k_{1}}\right) \lambda^{2}+E_{1} S_{1} v_{1}, \\
\alpha_{4}=\left(M_{2}+\frac{m_{2}\left(d_{2} \lambda+k_{2}\right)}{m_{2} \lambda^{2}+d_{2} \lambda+k_{2}}\right) \lambda^{2}+E_{4} S_{4} v_{4}, \\
\beta_{1}=\left(M_{1}+\frac{m_{1}\left(d_{1} \lambda+k_{1}\right)}{m_{1} \lambda^{2}+d_{1} \lambda+k_{1}}\right) \lambda^{2}-E_{1} S_{1} v_{1}, \\
\beta_{4}=\left(M_{2}+\frac{m_{2}\left(d_{2} \lambda+k_{2}\right)}{m_{2} \lambda^{2}+d_{2} \lambda+k_{2}}\right) \lambda^{2}-E_{4} S_{4} v_{4} .
\end{array}\right\}
$$

Using physical model parameters given in Tables 1 , 2 , and 5 , combined with deflection angle $\theta=0.4307^{\circ}$ and equations (8)-(17), the coefficients $\bar{A}_{i}$ and $\bar{B}_{i}$ in equation (17) are obtained by MATLAB, as shown in Table 6.

\section{Results and Discussion}

5.1. Influence of Ocean Current. In the process of deep-sea mining operation, the lifting pipe will be offset due to the action of ocean current load. When the parameters of the lifting pipe have been determined, the mass of the buffer $\left(M_{2}\right)$ and the force of the ocean current $\left(q_{N}\right)$ are the important factors affecting the deflection angle $(\theta)$. According to Morison equation, the current force is a function about the relative velocity of vessel and ocean current. In order to illustrate the effects of ocean current on the lifting pipe dynamic characteristics during deep-sea mining operation, seven buffer masses are selected with different sailing velocities $\left(V_{0}\right)$ of the mining vessel.

It is assumed that the mining vessel is considered to be dynamically stable and sailing against the current during towing. The comparison of the calculated results obtained from these states is conducted on aspects of deflection angle. As shown in Figure 9, under the condition of the same sailing velocity of the mining vessel, the deviation angle of the lifting pipe becomes smaller with the increase of the mass of buffer. Meanwhile, under the condition of the same buffer mass, the maximum deviation angle takes place at the maximum sailing velocity of the mining vessel; obviously the minimum deviation angle occurs when the sailing velocity is minimum. Moreover, the buffer mass has little effect on the deflection angle when the sailing velocity is smaller.

In order to intuitively understand the influence on the dynamic characteristics of the lifting pipe caused by the change of buffer mass and sailing velocity, the axial load and stress of the lifting pipe under aforementioned buffer mass and sailing velocity are simulated using the software OrcaFlex, as shown in Figure 10.

It can be seen from Figure 10 that the axial load decreases gradually with the increase of water depth. The axial load has a small abrupt change at $1000 \mathrm{~m}$ due to the influence of the mass of the pump unit. For the whole lifting pipe, the axial load is continuous and is equal at the joint of any two-stage pipe. As shown in equation (19), the axial stress equation is a piecewise function related to the cross-sectional area, which is discontinuous due to the stepped structure of the pipe, so the axial stress is discontinuous. The dynamic load of lifting pipe is higher than its static load under the same sea condition. The maximum axial load takes place at the top of the pipe, the maximum static stress takes place at the top or $1000 \mathrm{~m}$ of the lifting pipe, and the maximum axial dynamic stress takes place at $2000 \mathrm{~m}$ of the pipe.

As a whole, the axial static load significantly increases with an increase in buffer mass, and the maximum axial static load at the top of it attains $4.73 \times 106 \mathrm{~N}$ at $60 \mathrm{~T}$ and increases $14.25 \%$ compared to the calculated value $4.14 \times 106 \mathrm{~N}$ at $0 \mathrm{~T}$. The axial dynamic load of the lifting pipe 
TABLE 6: Values of $\bar{A}_{i}$ and $\bar{B}_{i}$ in equation (17) with DVA.

\begin{tabular}{lcccc}
\hline$i$ & 1 & 2 & 3 & 4 \\
\hline $\bar{A}_{i}$ & $1.3633-0.3881 j$ & $-0.0053-0.0269 j$ & $0.0002+0.0002 j$ & $-0.0003-0.0013 j$ \\
$\bar{B}_{i}$ & $0.1317+0.3881 j$ & $1.7061+4.7149 j$ & $0.8981+6.7834 j$ & $-2.9977+11.0151 j$ \\
\hline
\end{tabular}

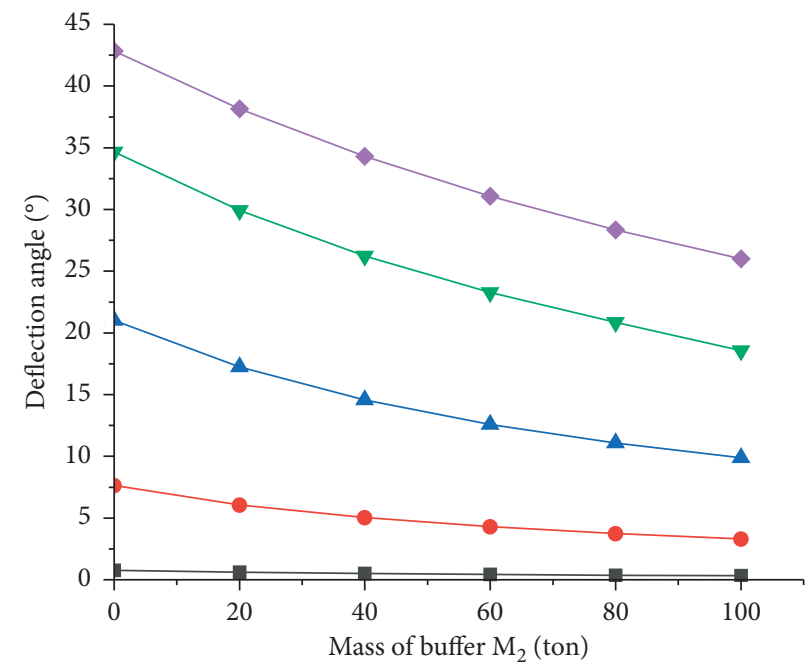

$$
\begin{aligned}
\rightarrow \mathrm{V}_{0}=0 \mathrm{~m} / \mathrm{s} & \rightarrow \mathrm{V}_{0}=1.2 \mathrm{~m} / \mathrm{s} \\
\rightarrow \mathrm{V}_{0}=0.4 \mathrm{~m} / \mathrm{s} & \multimap \mathrm{V}_{0}=1.5 \mathrm{~m} / \mathrm{s} \\
\neg \mathrm{V}_{0}=0.8 \mathrm{~m} / \mathrm{s} &
\end{aligned}
$$

Figure 9: Effect of buffer mass on deflection angle under different sailing velocities.

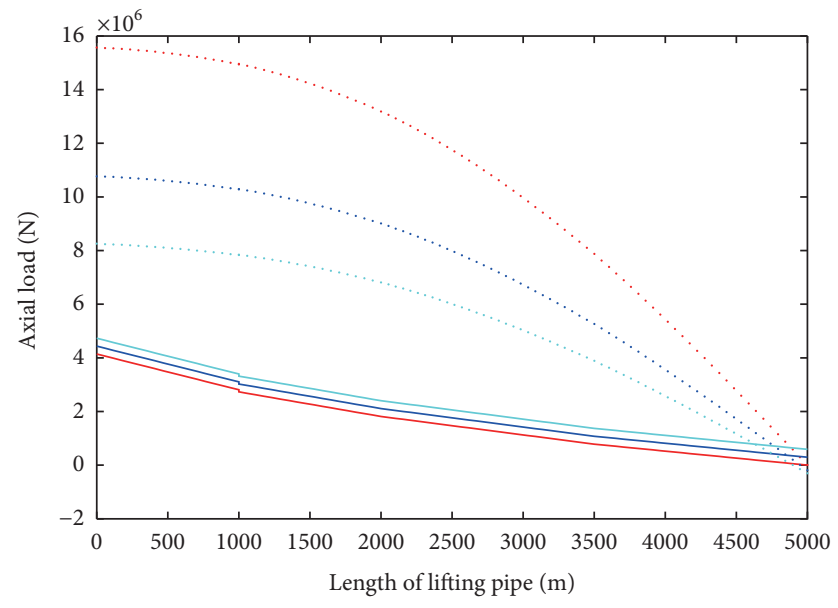

$\begin{array}{lll}\text { Gravity load } M_{2}=0 \text { ton } & \ldots . . . & \text { Dynamic load } M_{2}=30 \text { ton } \\ \text { Dynamic load } M_{2}=0 \text { ton } & \text { Gravity load } M_{2}=60 \text { ton } \\ \text { Gravity load } M_{2}=30 \text { ton } & \ldots . . . & \text { Dynamic load } M_{2}=60 \text { ton }\end{array}$

(a)

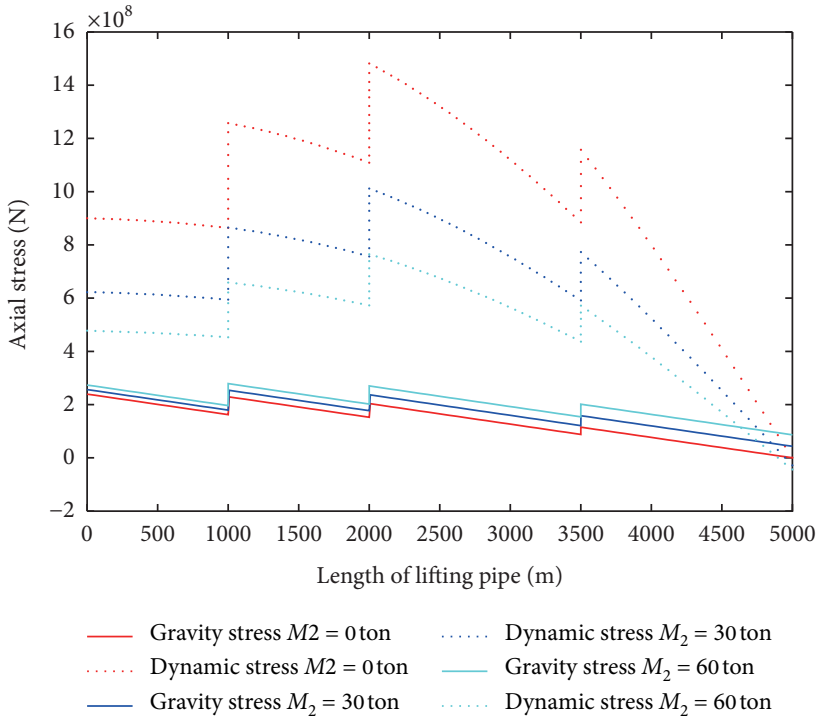

(b)

Figure 10: Continued. 


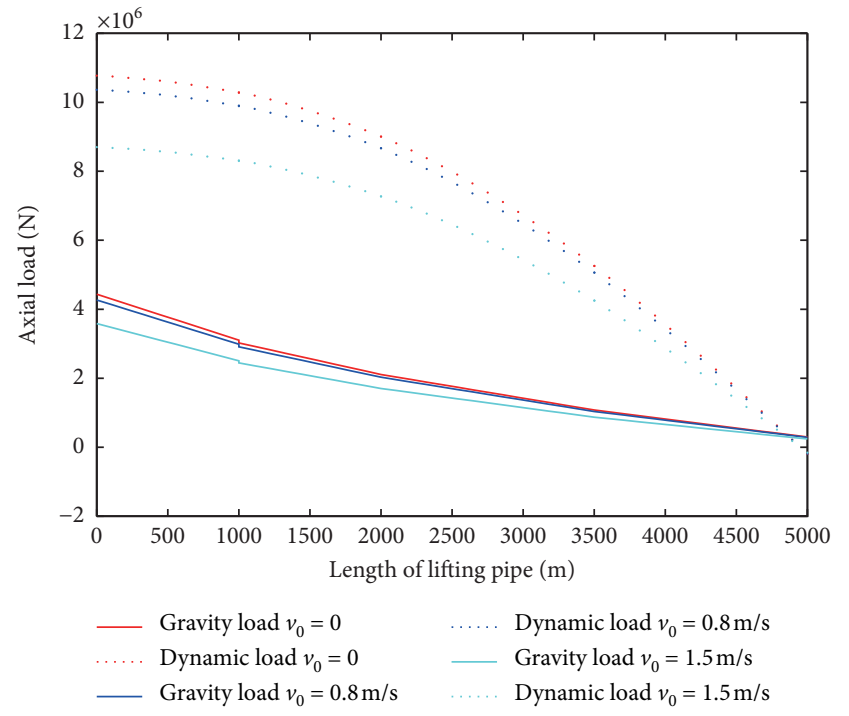

(c)

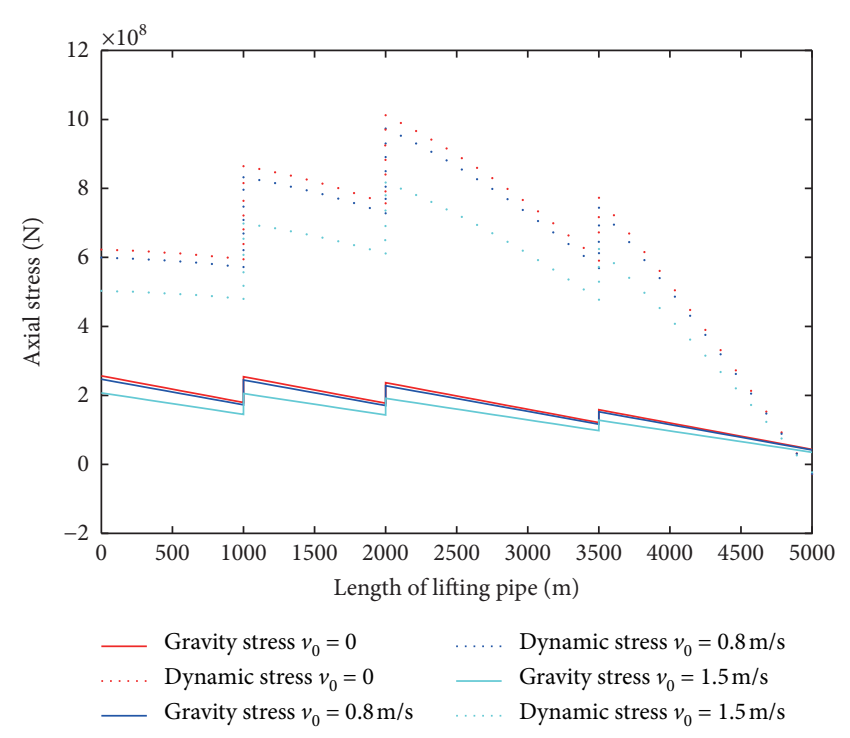

(d)

Figure 10: Axial load and stress of the lifting pipe. (a) Influence of buffer mass on axial load. (b) Influence of buffer mass on axial stress. (c) Influence of sailing velocity on axial load. (d) Influence of sailing velocity on axial stress.

decreases gradually with the increase of the buffer mass, and the maximum dynamic load at the top of it attains $15.6 \times 106 \mathrm{~N}$ at $0 \mathrm{~T}$ and increases $89.09 \%$ compared to the calculated value $8.25 \times 106 \mathrm{~N}$ at $60 \mathrm{~T}$. Similarly, the axial stress changes in the same way as the axial load; when the mass of buffer is $0 \mathrm{~T}$ and $60 \mathrm{~T}$, the maximum axial static stress is $2.39 \times 108 \mathrm{~Pa}$ and $2.79 \times 108 \mathrm{~Pa}$, respectively, with a difference of $16.74 \%$, and the maximum axial dynamic stress is $14.82 \times 108 \mathrm{~Pa}$ and $7.65 \times 108 \mathrm{~Pa}$, with a difference of $93.73 \%$. In addition, assuming that the mass of the buffer is a fixed value, the axial static load, axial dynamic load, axial static stress, and axial dynamic stress of the lifting pipe gradually decrease with an increase in the sailing velocity of the mining vessel.

Undoubtedly, the aforementioned results adequately demonstrate that obvious buffer mass and sailing velocity strongly influences not only lateral displacement of the lifting pipe, but also axial load and axial stress; among them, the influence of buffer mass on dynamic load and dynamic stress is more significant. Therefore, a reasonable selection of the buffer mass and the sailing velocity of the mining vessel in the practical engineering can effectively decrease the total axial load of the lifting pipe.

5.2. Comparison of the Lifting Pipe with and without DVA. In order to further explain the vibration characteristics of the lifting pipe after adding DVA, in this section, we compare the longitudinal vibration characteristics of the lifting pipe with and without DVA. Figure 11 is the comparison of the lifting pipe's longitudinal vibration with and without DVA on time $=0$.
It can be seen from Figure 11 that when the DVAs are attached at this subsystem, the longitudinal vibration amplitude is greatly reduced with the increase of the lifting pipe's length. At $5000 \mathrm{~m}$, the longitudinal vibration amplitude of the subsystem with DVAs is $13.24 \mathrm{~m}$, while that of the subsystem without DVA is $16.75 \mathrm{~m}$. After attaching DVAs, the longitudinal amplitude is reduced by $20.9 \%$.

Figure 12 is the longitudinal displacements of the lifting pipe with DVAs from 0 to 20 seconds. Form it, we can see that after attaching DVAs the vibration frequency of the lifting pipe is not changed, it still vibrates with the motion period of sea wave, and the vibration displacement amplitude is reduced when DVAs are installed.

When the DVAs is equipped, the deflection angle of the lifting pipe subsystem decreases from $0.5709^{\circ}$ to $0.4307^{\circ}$ under the action of sea waves, and the lateral displacement of the lifting pipe is reduced from 49.8196 to 37.5853 . So, after equipping DVAs, the lateral displacement at the end of the pipe is reduced by $24.56 \%$.

5.3. Comparison of Axial Force and Stress. In this section, we compare the axial force and stress characteristics of the lifting pipe with and without DVA. The MATLAB software was used to calculate equations (19)-(20), and the simulation results about gravity stress, dynamic stress, and total axial stress of the pipe with and without DVA are shown in Figures 13-16.

It can be seen from Figure 13 that the dynamic load is larger than the gravity load, and the trend is consistent with the total load. Figures 14-16 show a comparison between with and without dynamic vibration absorber. For gravity 


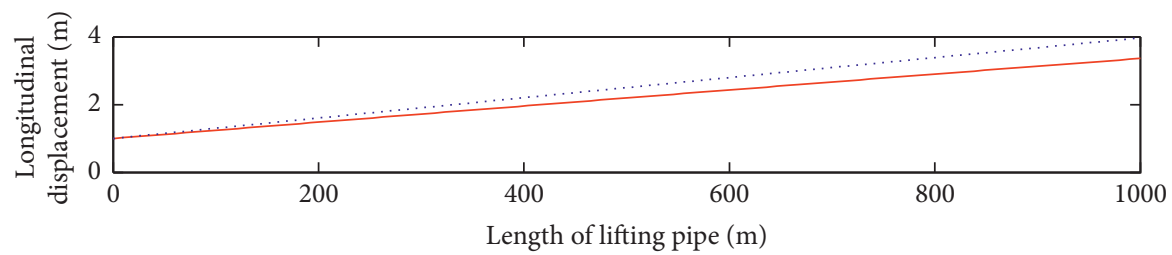

With DVA

Without DVA

(a)

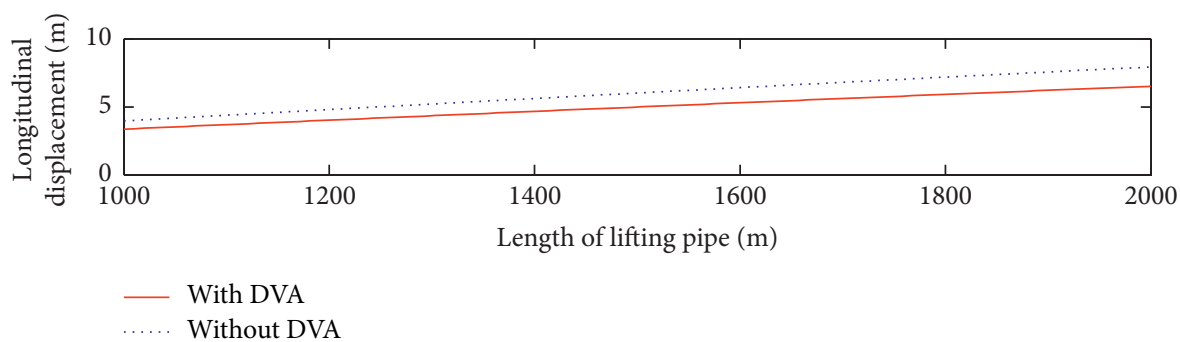

(b)

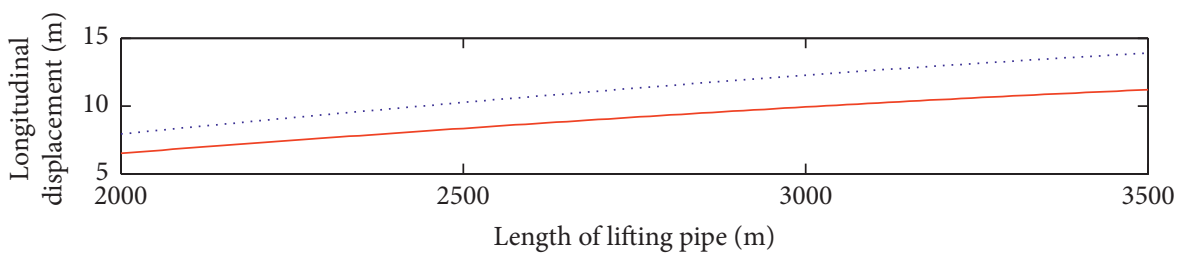

With DVA

Without DVA

(c)

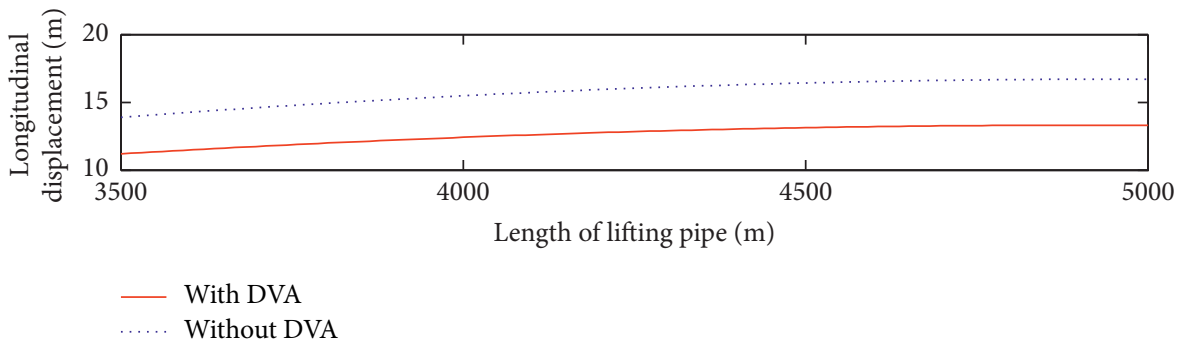

(d)

FIGURE 11: Comparison of the lifting pipe's longitudinal vibration with and without DVA. (a) $x: 0 \longrightarrow 1000 \mathrm{~m}$. (b) $x: 1000 \longrightarrow 2000 \mathrm{~m}$. (c) $x$ : $2000 \longrightarrow 3500 \mathrm{~m}$. (d) $x: 3500 \longrightarrow 5000 \mathrm{~m}$.

load and gravity stress given in Figure 14, no matter with or without DVA, the downward trend of gravity load or gravity stress does not change but their values increase. At the top of the lifting pipe, the gravity stress increases from $0.261 \mathrm{GPa}$ to $0.279 \mathrm{GPa}$. For dynamic load and dynamic stress, attaching DVAs can reduce the dynamic load and dynamic stress, as shown in Figure 15. And it is obvious that, at the top of the pipe, the dynamic stress is $0.624 \mathrm{GPa}$ without DVA, and it decreases to $0.501 \mathrm{GPa}$ when attaching DVAs. For the total axial load and axial stress, the same effect can also be observed from Figure 16 where the value of total axial stress of the lifting pipe with DVAs is $0.786 \mathrm{GPa}$ at the top, and the value reaches $0.887 \mathrm{GPa}$ without DVA. Therefore, attaching DVAs can effectively reduce the axial stress of the lifting pipe.

Using the experimental platform built in Figure 1, the experiment axial force of the connection point between the vessel and the lifting pipe is obtained. The simulation axial 


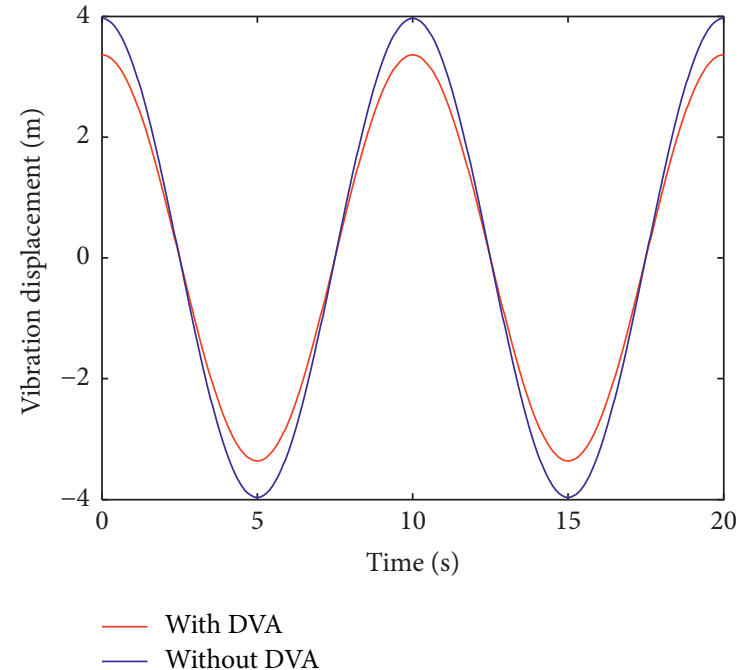

(a)

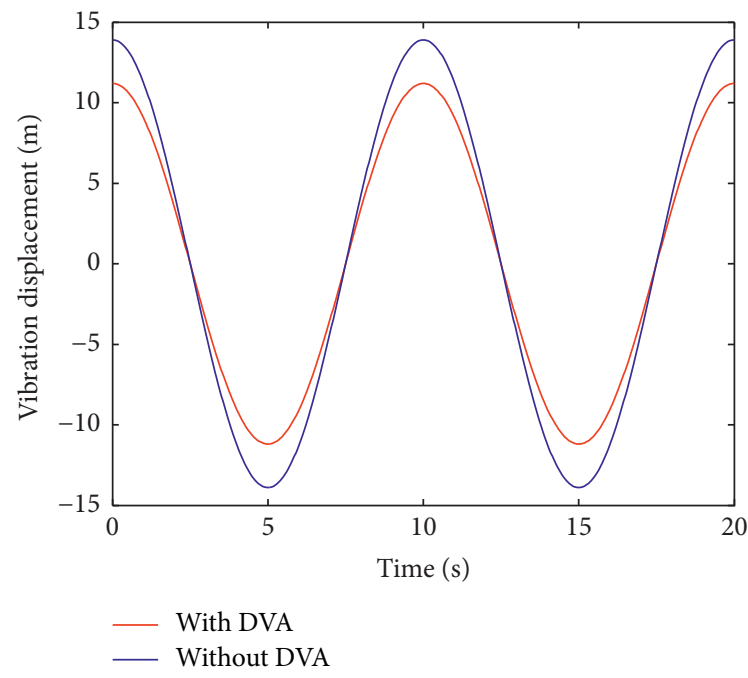

(c)

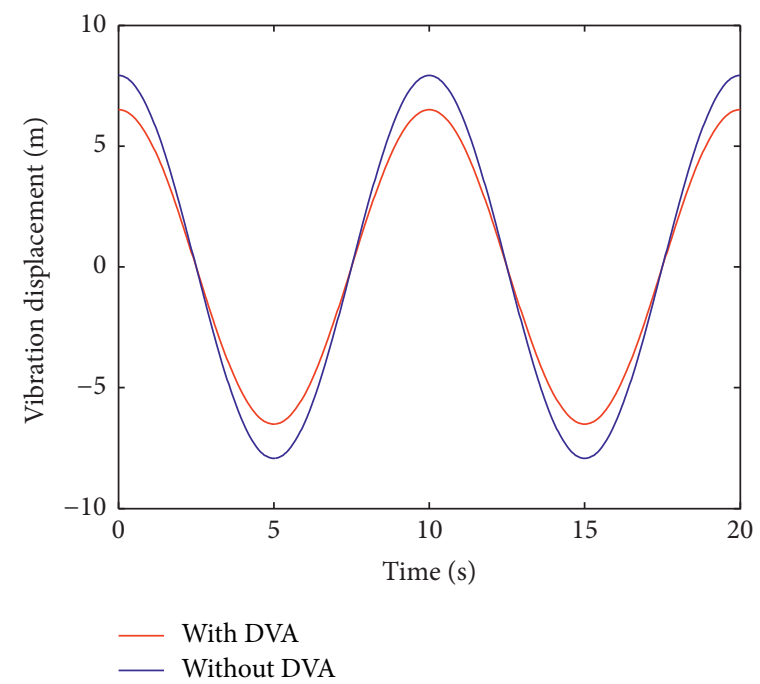

(b)

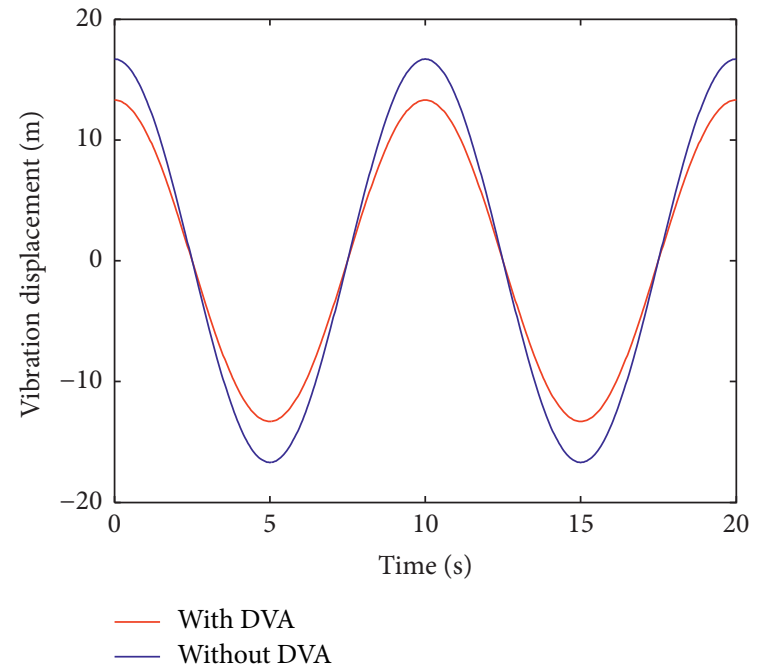

(d)

Figure 12: Longitudinal displacements of the lifting pipe. (a) $x=1000 \mathrm{~m}$. (b) $x=2000 \mathrm{~m}$. (c) $x=3500 \mathrm{~m}$. (d) $x=5000 \mathrm{~m}$.

force can be calculated by MATLAB, and the experimental value and simulation value are compared to verify the usefulness and reliability of the mathematical model proposed. The data comparison is shown in Figure 17.
It can be seen from Figure 17 that the experimental curve is very consistent with the simulation curve. It can be claimed that this difference may be due to the axial force collection error and the difference 


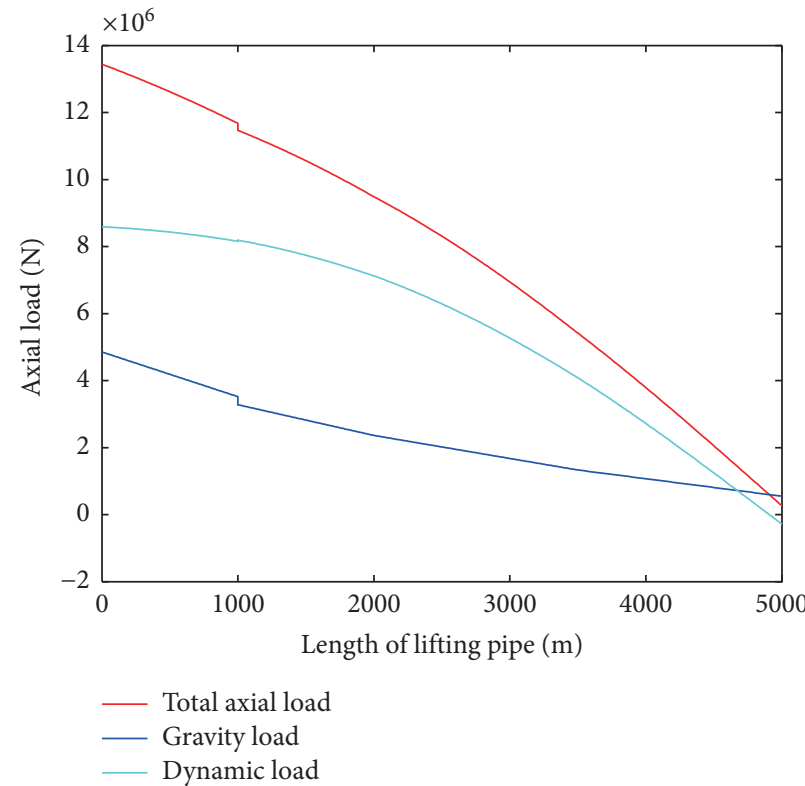

(a)

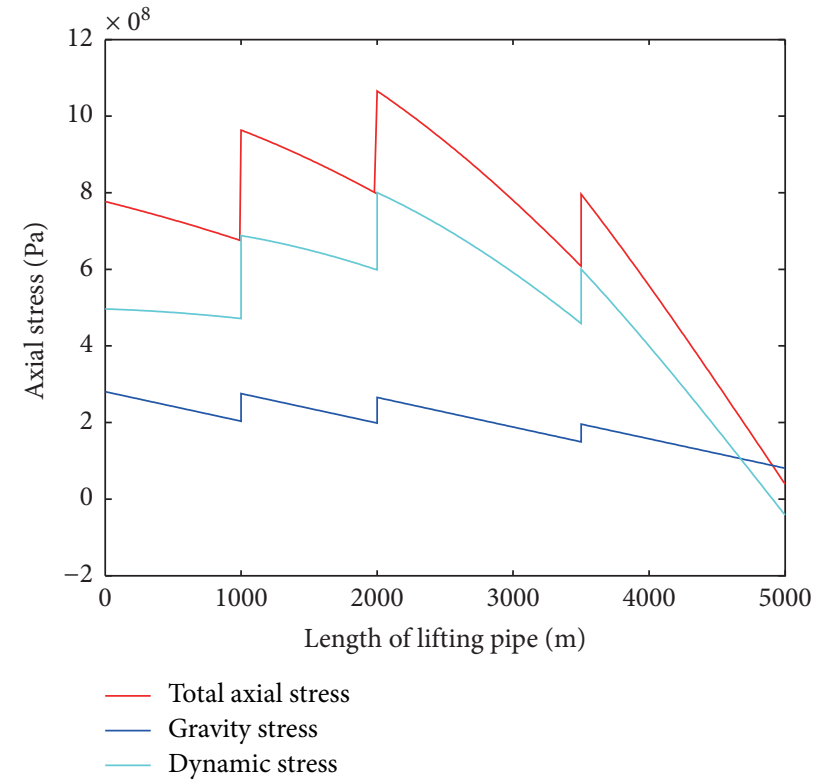

(b)

Figure 13: Axial load and stress with DVA. (a) Axial load. (b) Axial stress.

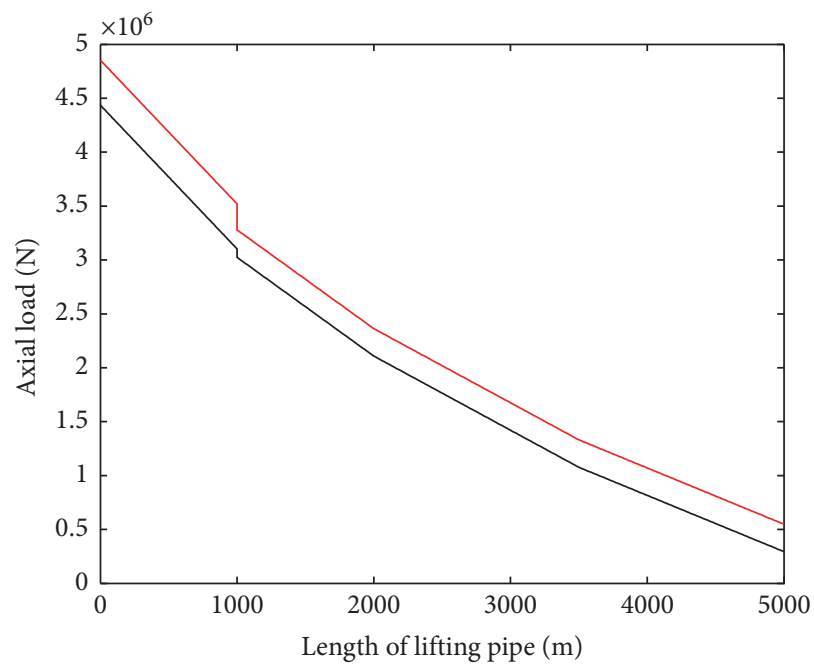

Without DVA

With DVA

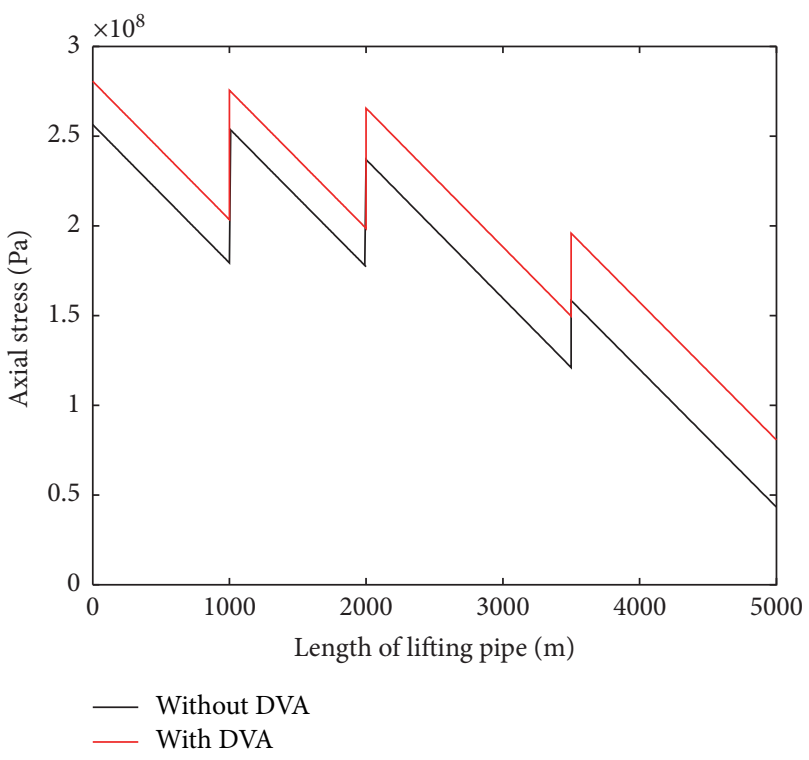

(b)

Figure 14: Gravity load and stress with/without DVA. (a) Axial load. (b) Axial stress. 


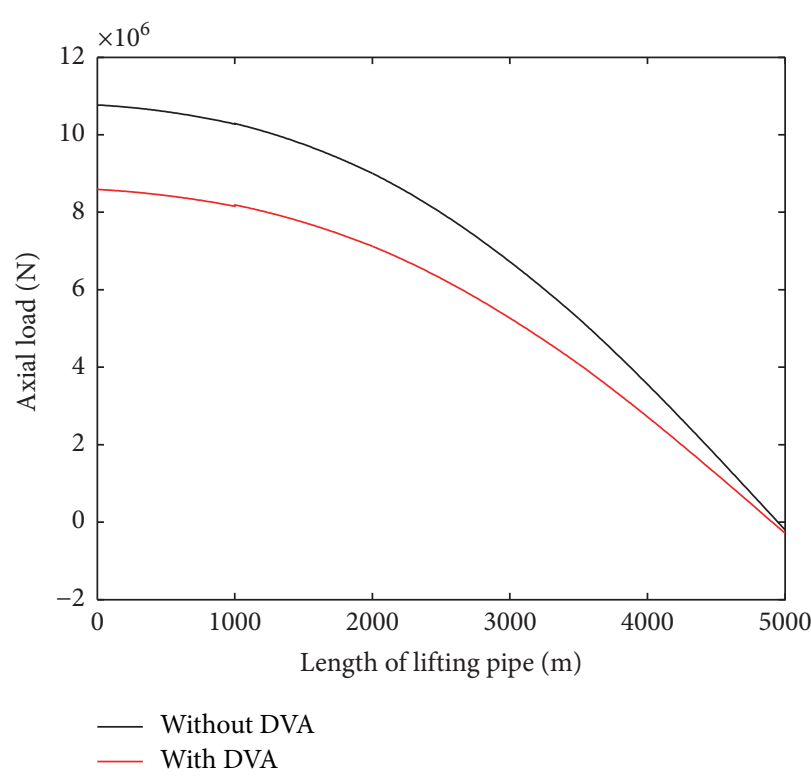

(a)

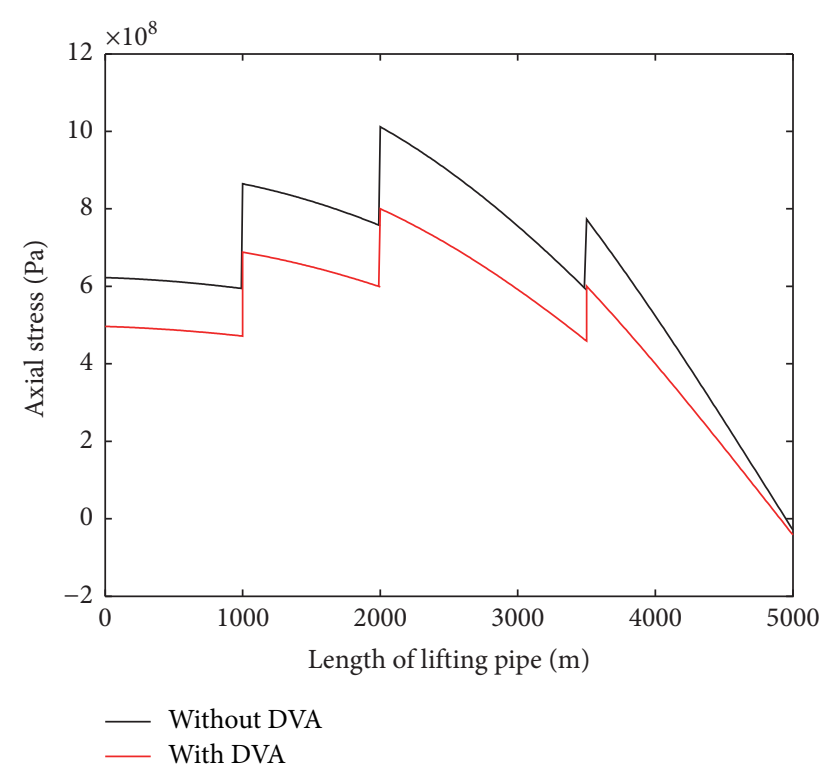

(b)

Figure 15: Dynamic load and stress with/without DVA. (a) Axial load. (b) Axial stress.

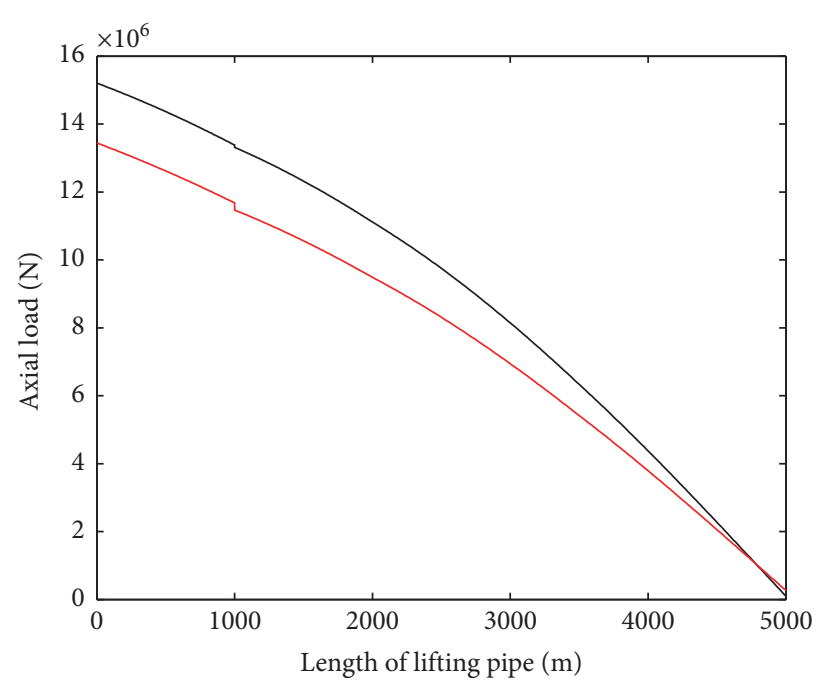

Without DVA

With DVA

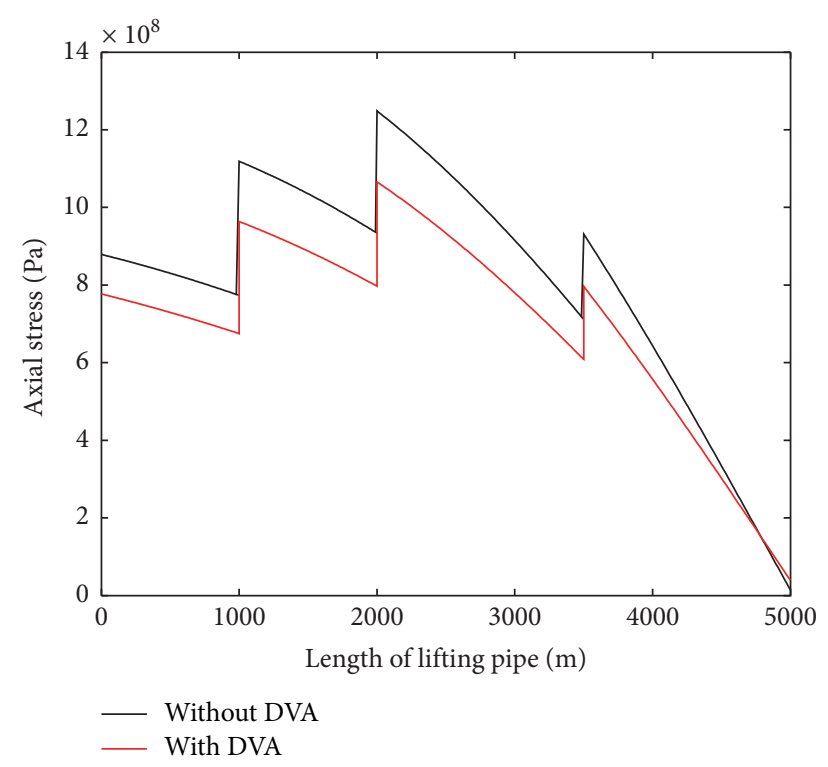

(b)

Figure 16: Total axial load and stress with/without DVA. (a) Axial load. (b) Axial stress. 


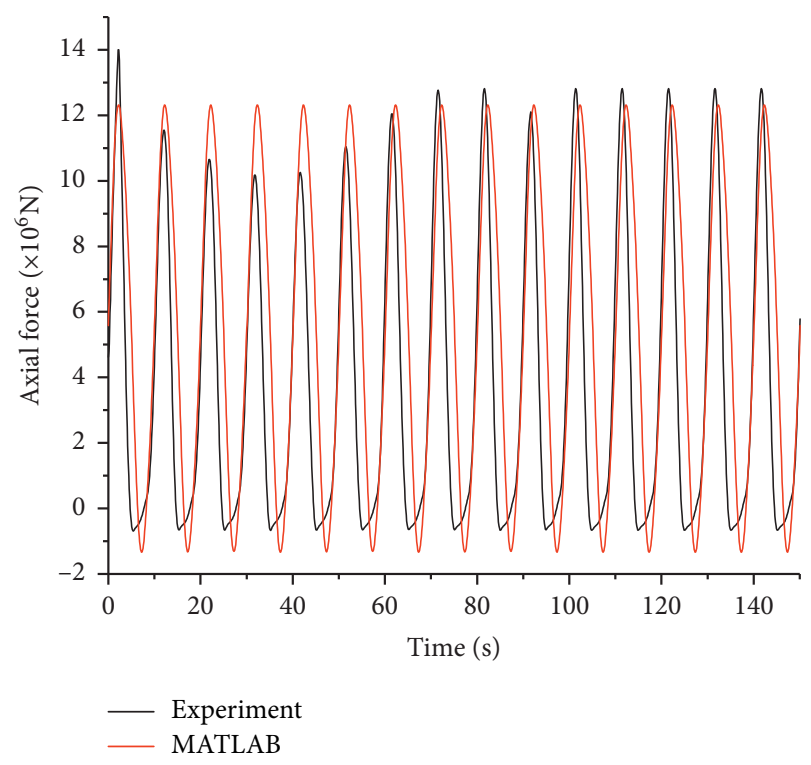

FIGURE 17: Comparison of experiment and simulation.

between the physical model and the prototype of the lifting system.

\section{Conclusion}

The main goal of the proposed model is to allow for more accurate dynamic model considering the influence of ocean current with DVAs in typical lifting pipe of deepsea mining. Considering the geometrical features of the prototype, the physical model of lifting system is built at the National Ocean Technology Center of China, which simulates different marine environmental loads. Combined with the linearization method and current velocity, the equation of moment balance is derived, and then the deflection angle of lifting pipe is calculated by MATLAB. At the same time, a comprehensive deep-sea mining model is established by using Orcaflex software, and the influence of the buffer mass and sailing velocity on the deflection angle and the axial load and stress of the lifting pipe is studied. Applying the method of separation of variables, exact analytical solutions of the vibration and axial stress excited by the heave motion of mining vessel are determined with and without DVAs. The vibration process is modeled in MATLAB and Orcaflex to assess its feasibility, and the comparative analysis has shown that the proposed mathematical model of lifting pipe can be useful and reliable.

Since the solution of vibration and axial stress of the system equipped with DVAs is obtained according only to the experimental model in this work, the parameters combination of dynamic vibration absorbers cannot be directly used in production due to the different working environment of deep-sea mining. But this method has important guiding significance for the vibration and axial stress analysis of lifting pipe of deep-sea mining.

\section{Data Availability}

The data used to support the findings of this study are available from the corresponding author upon request.

\section{Conflicts of Interest}

The authors declare that there are no conflicts of interest regarding the publication of this paper.

\section{Acknowledgments}

This work was supported by the National Natural Science Foundation of China (51774193), Shandong Provincial Natural Science Foundation (ZR2020MF101), Shandong Province Key Laboratory of Mine Mechanical Engineering Open Fund (2019KLMM202), and SDUST Research Fund (2018TDJH101).

\section{References}

[1] S. H. Ali, D. Giurco, N. Arndt et al., "Mineral supply for sustainable development requires resource governance," Nature, vol. 543, no. 7645, pp. 367-372, 2017.

[2] C. John, "Extraction in four dimensions: time, space and the emerging Geo(-)politics of deep-sea mining," Geopolitics, vol. 25, no. 1, pp. 1-25, 2020.

[3] K. A. Miller, K. F. Thompson, J. Paul, and D. Santillo, “An overview of seabed mining including the current state of development, environmental impacts, and knowledge gaps," Frontiers in Marine Science, vol. 4, pp. 1-24, 2018.

[4] J. Thorsen, N. R. Challabotla, S. Saevik, and O. J. Nydal, "A numerical study on vortex-induced vibrations and the effect of slurry density variations on fatigue of ocean mining risers," Ocean Engineering, vol. 174, pp. 1-13, 2019.

[5] J. R. Hein, K. Mizell, A. Koschinsky, and T. A. Conrad, "Deepocean mineral deposits as a source of critical metals for highand green-technology applications: comparison with landbased resources," Ore Geology Reviews, vol. 51, pp. 1-14, 2013.

[6] S. G. Cho, S. Park, J. Oh et al., "Design optimization of deepseabed pilot miner system with coupled relations between constraints," Journal of Terramechanics, vol. 83, pp. 25-34, 2019.

[7] Y. Dai, X. Y. Li, W. W. Yin, Z. H. Huang, and Y. Xie, "Dynamics analysis of deep-sea mining pipeline system considering both internal and external flow," Marine Georesources \& Geotechnology, vol. 39, no. 4, pp. 408-418, 2019.

[8] K. Aso, K. Kan, H. Doki et al., "Effects of vibration absorbers on the longitudinal vibration of a pipe string in the deep seapart 1: in case of mining cobalt crust," International Journal of Offshore and Polar Engineering, vol. 2, pp. 309-317, 1992.

[9] K. Aso, K. Kan, H. Doki et al., "The effects of vibration absorbers on the longitudinal vibration of a pipe string in the deep sea-part 2: a case of mining manganese nodules," International Journal of Offshore and Polar Engineering, vol. 4, pp. 62-67, 1994.

[10] K. Aso, K. Kan, H. Doki et al., "A method for semi-active control of the longitudinal vibration of a pipe string for mining manganese nodules," in Proceedings of the Fourth International Offshore and Polar Engineering Conference Osaka, pp. 301-306, Osaka, Japan, April, 1994. 
[11] Y. Kobayashi and K. Aso, "Longitudinal vibration-control of pipe string for mining manganese nodules in deep sea with an elastic support," in Proceedings of the Seventh International Offshore and Polar Engineering Conference, pp. 25-30, Honolulu, HI, USA, May 1997.

[12] G. Cui and K. Aso, "The optimum design of a stepped pipe string with vibration absorbers for mining manganese nodules in the deep sea with consideration for reducing its maximum axial stress," in Proceedings of the 16 International Conference on Offshore Mechanics and Arctic Engineering, pp. 315-322, Newyork, NY, USA, May 1997.

[13] H. Erol, "Vibration analysis of stepped-pipe strings for mining from deep-sea floors," Ocean Engineering, vol. 32, pp. 37-55, 2005.

[14] Y. Dai and S. J. Liu, "An integrated dynamic model of ocean mining system and fast simulation, of its longitudinal reciprocating motion," China Ocean Engineering, vol. 27, no. 2, pp. 231-244, 2013.

[15] J. W. Oh, C. H. Lee, S. Hong, D. S. Bae, H. J. Cho, and H. W. Kim, "A study of the kinematic characteristic of a coupling device between the buffer system and the flexible pipe of a deep-seabed mining syste," International Journal of Naval Architecture and Ocean Engineering, vol. 6, pp. 652669, 2014.

[16] S. S. Kim, H. S. Yun, C. H. Lee, H. W. Kim, and S. Hong, "Efficient analysis of a deep-seabed integrated mining system using a subsystem synthesis method," in Proceedings of the Asme International Design Engineering Technical Conferences \& Computers \& Information in Engineering Conference, pp. 1-6, Boston, MA, USA, August 2015.

[17] C. H. Lee, S. Hong, H. W. Kim, and S.-S. Kim, "A comparative study on effective dynamic modeling methods for flexible pipe," Journal of Mechanical Science and Technology, vol. 29, no. 7, pp. 2721-2727, 2015.

[18] W. Ma, D. Schott, and G. Lodewijks, "A new procedure for deep sea mining tailings disposal," Journal of Offshore Mechanics and Arctic Engineering, vol. 139, pp. 1-9, 2017.

[19] H. Yang and S. J. Liu, "Measuring method of solid-liquid twophase flow in slurry pipeline for deep-sea Mining,Thalassas," International Journal of Marine Science, vol. 34, no. 2, pp. 459-469, 2018.

[20] H. Yang and S. J. Liu, "Heave compensation system of lifting pipeline for deep-sea mining based on combined action of vibration absorber and accumulator," Marine Georesources \& Geotechnology, vol. 37, no. 3, pp. 393-401, 2019.

[21] Y. W. Li, S. J. Liu, and X. Z. Hu, "Research on rotating speed's influence on performance of deep-sea lifting motor pump based on DEM-CFD," Marine Georesources \& Geotechnology, vol. 37, no. 8, pp. 979-988, 2019.

[22] S. J. Liu, H. Wen, W. S. Zou, X. Z. Hu, and Z. Dong, "Deep-sea mining pump wear prediction using numerical two-phase flow simulation," in Proceedings of the International Conference on Intelligent Transportation, Big Data \& Smart City (ICITBS), pp. 630-636, Changsha, China, January 2019.

[23] Q. Liu and L. J. Xiao, "Analysis and research on longitudinal vibration characteristics of deep sea mining pipe based on finite element method," Mathematical Problems in Engineering, vol. 2020, Article ID 8219794, 18 pages, 2020.

[24] L. J. Xiao, Q. Liu, and J. M. Lu, "Analysis on longitudinal vibration law of deep sea stepped mining pipe," AIP Advances, vol. 10, no. 12, Article ID 125302, 2020.

[25] Q. H. Song, H. Y. Jiang, Q. J. Song, L. Xiao, and Q. Liu, "Longitudinal vibration compensation model of stepped-pipe strings in deep-sea mining," PLos One, vol. 15, no. 11, Article ID e0241650, 2020.

[26] J. S. Chung and C. A. Felippa, "Nonlinear static analysis of deep ocean mining pipe-Part II: numerical studies," Journal of Energy Resources Technology, vol. 103, no. 1, pp. 16-25, 1981.

[27] B. Zhang, Z. Q. Shang, T. Wang, and Z. Wang, "Hydrodynamic load analysis and experimental study of grouting clamp under wave and ocean current," Journal of Marine Science and Engineering, vol. 8, no. 7, p. 512, 2020. 